\title{
Interface effects on the magnetic-proximity-induced quantized Hall response in heterostructures based on three-dimensional topological insulators
}

\author{
V. N. Men'shov, ${ }^{1,2,3,4}$ I. A. Shvets, ${ }^{3,4}$ and E. V. Chulkov ${ }^{1,4,5}$ \\ ${ }^{1}$ Donostia International Physics Center (DIPC), P. de Manuel Lardizabal 4, 20018 San Sebastián, Basque Country, Spain \\ ${ }^{2}$ NRC Kurchatov Institute, Kurchatov Sqr. 1, 123182 Moscow, Russia \\ ${ }^{3}$ Tomsk State University, 634050 Tomsk, Russia \\ ${ }^{4}$ Saint Petersburg State University, Saint Petersburg 198504, Russia \\ ${ }^{5}$ Departamento de Física de Materiales UPV/EHU, CFM-MPC UPV/EHU, 20080 San Sebastián/Donostia, Basque Country, Spain
}

(Received 17 October 2018; published 4 March 2019)

\begin{abstract}
We report a theoretical study of the spin-dependent transport properties in heterostructures containing a three-dimensional topological insulator (TI) thin film and ferromagnetic normal insulator (FMNI) slab. Within the framework of a continual approach for the FMNI/TI/FMNI trilayer model, we reveal how the magnetic proximity effect at the TI/FMNI interface can influence an intrinsic Hall response of the system. We predict that the FMNI/TI/FMNI trilayer undergoes a transition into the quantum anomalous Hall phase either from the topologically trivial phase or from the quantum spin Hall phase, which is controlled by tuning the proximity-induced exchange field, the TI film thickness, and the TI band structure parameters. We draw the corresponding phase diagram of the FMNI/TI/FMNI trilayer. Moreover, we argue that the roughness at the TI/FMNI interfaces can cause the decomposition of the TI film into topologically distinct domains, which affects the Hall conductivity. We discuss the specifics of manifestation and complexities of observation of quantized conductivity in realistic TI/FMNI heterostructures.
\end{abstract}

DOI: 10.1103/PhysRevB.99.115301

\section{INTRODUCTION}

New classes of materials manifesting topological phases offer a versatile platform for the fundamental science studies of condensed matter as well as new paradigms for the design of devices providing the next generation of wide-ranging applications in spintronics [1]. Numerous studies have focused on understanding topological insulators (TIs) and phenomena they exhibit such as quantum spin Hall effect (QSHE) [2-4], quantum anomalous Hall effect (QAHE) [3-9], and intrinsic magnetoelectric effect $[3,4,10]$. The search for materials and complex systems capable of demonstrating QAHE at elevated temperatures is restricted by the requirement of simultaneous existence of the nontrivial Berry curvature [11] and an exchange interaction that breaks the time-reversal symmetry in TI. Both experimental and theoretical investigations in this important field are far from being exhaustive.

The most straightforward approach to bring a long-range magnetic order in TI and achieve the QAHE regime is to dope it with magnetic atoms [6-9,12-18]. The narrow-gap tetradymite-type semiconductors $A_{2} B_{3}$, where $A=\mathrm{Bi}$, Sb and $B=\mathrm{Se}, \mathrm{Te}$, are prototypical three-dimensional (3D) TIs displaying strong enough spin-orbit coupling (SOC) to produce band inversion. The studies of dilute magnetic 3D TIs with a small percentage of transition metal atoms have shown the spontaneous long-range ferromagnetic (FM) ordering of dopant's magnetic moments usually appearing at the rather low Curie temperature $T_{C}[6]$. The magnetotransport measurements [12-16] have been reported on the QAHE observation in $\mathrm{Cr}_{x}\left(\mathrm{Bi}_{1-y} \mathrm{Sb}_{y}\right)_{2-x} \mathrm{Te}_{3}$ and $\mathrm{V}_{x}\left(\mathrm{Bi}_{1-y} \mathrm{Sb}_{y}\right)_{2-x} \mathrm{Te}_{3}$ thin films. However, the Hall resistivity close to or at the perfectly quantized value has only been registered at cryogenic temperatures, which seriously impedes the progress in the field of the quantized spin transport. More recently, Mogi et al. [19] were successful at elevating temperature of QAHE by applying the digital alloy technique of epitaxial growth. They have fabricated the structures in which the FM quintuple layers (QLs) of $\mathrm{Cr}_{x}\left(\mathrm{Bi}_{1-y} \mathrm{Sb}_{y}\right)_{2-x} \mathrm{Te}_{3}$ with relatively high concentration of transition metal atoms $x=0.46$ were inserted close to the top and bottom interfaces of the TI $\left(\mathrm{Bi}_{1-y} \mathrm{Sb}_{y}\right)_{2} \mathrm{Te}_{3}$ film. Such selective modulation doping made it possible to stabilize QAHE up to $1 \mathrm{~K}$ (and even up to a few Kelvin [20]), which is two orders of magnitude higher than that for the uniformly doped film $\mathrm{Cr}_{x}\left(\mathrm{Bi}_{1-y} \mathrm{Sb}_{y}\right)_{2-x} \mathrm{Te}_{3}$ with $x=0.10$ [19].

Assuredly, in practice it is very difficult to regulate the magnetic impurity doping in the TI samples to produce the sufficiently strong exchange field while preserving the topological order unchanged. Indeed, raising both exchange field and $T_{C}$ requires increasing the doping level, which inevitably lowers the TI sample quality, and even destroys the topological order due to weakening SOC. A less invasive and more vigorous method to introduce the FM order into TI is the use of the magnetic proximity effect. When TI is closely neighbored to a material exhibiting FM or ferrimagnetic ordering, the spin-polarized states of the magnetic material overlap with the states of the TI material, which leads to an induced magnetization inside the TI near the interface.

The proximity-induced out-of-plane spin polarization of electrons in TIs was experimentally probed in a number of the epitaxial heterostructures including FMNIs (here and further, the abbreviation FMNI is referred to normal, i.e., topologically trivial, insulator, NI, with FM or ferrimagnetic 
ordering). Among a large number of TI/FMNI combinations, the $\mathrm{Bi}_{2} \mathrm{Se}_{3} / \mathrm{EuS}$ system (where $\mathrm{EuS}$ is a low-temperature insulating ferromagnetic) has been intensely studied and discussed in literature in the context of the extraordinary magnetic properties produced by the interface exchange coupling [21-27]. The FM transition metal trichalcogenide $\mathrm{Cr}_{2} \mathrm{Ge}_{2} \mathrm{Te}_{6}$ is a suitable FM substrate for epitaxial growth of $\mathrm{TI} \mathrm{Bi}_{2} \mathrm{Te}_{3}$ with a high-quality interface providing the spin polarization of the TI state $[28,29]$. The induced perpendicular magnetization is demonstrated to persist well above $400 \mathrm{~K}$ in a five-QL $\left(\mathrm{Bi}_{y} \mathrm{Sb}_{1-y}\right)_{2} \mathrm{Te}_{3}$ film fabricated atop a high-temperature (with $\left.T_{C} \simeq 560 \mathrm{~K}\right)$ ferrimagnetic insulator $\mathrm{Tm}_{3} \mathrm{Fe}_{5} \mathrm{O}_{12}$ [30]. The proximity effect was also experimentally probed in $\mathrm{Bi}_{2} \mathrm{Se}_{3}$, by using a thin film of high- $T_{C}$ ferrimagnetic insulator with large perpendicular anisotropy $\mathrm{BaFe}_{12} \mathrm{O}_{19}$ [31], and in system $(\mathrm{Ga}, \mathrm{Mn}) \mathrm{As} /(\mathrm{Bi}, \mathrm{Sb})_{2}(\mathrm{Te}, \mathrm{Se})_{3}$ including dilute ferromagnetic semiconductor [32].

The artificially designed heterostructure setups combining TIs with FMNIs can become a suitable platform for realizing QAHE, by utilizing, on the one hand, the advantage of a strong FM material to provide the elevated Curie temperature and large uniform spontaneous net magnetization in the perpendicular direction. On the other hand, the use of an appropriate wide-gap insulating material as a magnetic constituent in the TI/FMNI heterostructure allows for keeping the topological character of electron states near the interfaces from breakdown as well as avoiding the parasitic conducting channels bypassing these states. Conceptually, the intrinsic Hall response of 3D TI is determined by the Dirac-type fermion helical states with linear spectrum at the TI boundaries [33,34]. However, there is still a large gap in the theoretical description of the proximity-induced QAHE in the TI/FMNI heterostructure, and important aspects of this phenomenon remain to be clarified. Specifically, how do the electrostatic and exchange effects at the TI/FMNI interface affect electron states in thin TI films? Furthermore, how can an imperfection of real heterostructure such as the interface roughness influence the Hall conductivity? The focus of this work is to catch and describe analytically the role of these effects in the low-energy electron states in the FMNI/TI/FMNI trilayer and elucidate the conditions for the QAHE realization, taking into account peculiarities of the band structure of the 3D TI material.

The rest of the paper is organized as follows. In Sec. II, within the framework of the continuum scheme, we formulate an approach based on the formalism of effective spindependent interface potential (IP) to study electron properties of the heterostructure composed of films of 3D TI and 3D FMNI. In Sec. III, introducing a proper boundary problem at the interfaces, we explore the model of the FMNI/TI/FMNI trilayer with the ideally plane interfaces. We derive the effective 2D Hamiltonian for the trilayer and thoroughly analyze the dependence of its parameters on the IP strength, the film thickness, and the TI material characteristics. In Sec. IV, we demonstrate that, given the proximity-induced exchange splitting, a series of quantum transitions between the trivial band insulator and the QSHE and QAHE insulating phases can be driven by tuning the TI film thickness. At this point, we draw the corresponding phase diagram of the trilayer in detail. Also, we thoroughly describe the evolution of the spectrum and Berry curvature with the TI film thickness and the exchange splitting. In Sec. V we consider how the spatial fluctuations of the TI film thickness can influence the intrinsic Hall conductivity in the trilayer. In the context of the theoretical findings, we discuss the transport properties of the FMNI/TI/FMNI trilayer in the presence of the interface roughness. In the last section (Sec. VI), we summarize the main results of the work.

\section{A MODEL OF HETEROSTRUCTURE COMPOSED OF TI AND FMNI}

We study the heterostructure assembled from 3D TI films and FMNI ones. The constituents of the heterostructure are closely adjacent to each other, forming the TI/FMNI interface. Certainly, a band structure of such a system is very complicated: it contains the multitude of the Bloch electron states of different origins. For a concrete heterostructure, a full picture of the band states can be obtained by means of numerical simulations, for instance, using density functional theory (DFT) calculations. Fortunately, it turns out to be sufficient taking into consideration only a small relevant set of the states from the whole multitude to qualitatively analyze some phenomena of particular interest. For example, when the heterostructure consists of a wide-gap semiconductor as the FMNI and a narrow-gap semiconductor as the TI, so that the TI bulk gap is entirely inside the FMNI bulk gap, one can pick out a set of low-energy states of the TI film, which are placed at the Fermi level and well separated in energy from the states related to the FMNI film. Then, electrons on the TI side of the interface carry mostly the prominent features associated with the topological order. It is also desirable for the topologically relevant states to be far away in energy from trivial Schokley-type states that often appear at the semiconductor interfaces due to band bending. Under these stipulations, one can construct a relatively simple model kp Hamiltonian $\mathbb{H}(-i \nabla)$ on the basis of the low-energy states of the 3D TI bulk material. At the same time, without going into details of the wave-function behavior out of the TI film, one can reproduce the effect of the TI/FMNI interface on the electron states inside the TI film additionally introducing a confining potential $\mathbb{W}(\mathbf{r}), \mathbf{r}=(x, y, z)$. Following, we use this approach to describe the spin-dependent transport in the TI/FMNI heterostructure. To be specific, in what follows, we focus on a FMNI/TI/FMNI trilayer consisting of a 3D TI film sandwiched by the FMNI slabs, which can be viewed as an elemental building block of the TI/FMNI heterostructure. The energy of the relevant electron states in the confined TI film generally reads as

$$
\Omega=\int d \mathbf{r} \Theta^{\dagger}(\mathbf{r})[\mathbb{H}(-i \nabla)+\mathbb{W}(\mathbf{r})] \Theta(\mathbf{r}),
$$

where $\Theta(\mathbf{r})$ is electron envelope function $(\mathrm{EF})$.

We utilize a generally accepted kp scheme (see, for instance, Refs. [35,36]) for describing the low-energy and long-wavelength bulk states of the narrow-gap semiconductor materials of $\mathrm{Bi}_{2} \mathrm{Se}_{3}$ type. It is constructed on the basis $u_{\Gamma}=\{|+\uparrow\rangle,|-\uparrow\rangle,|+\downarrow\rangle,|-\downarrow\rangle\}$, formed by four hybridized states of the $\mathrm{Se}$ and $\mathrm{Bi} p_{z}$ orbitals with the indices $+/-$ and $\uparrow / \downarrow$ that stand for even/odd parity and spin 


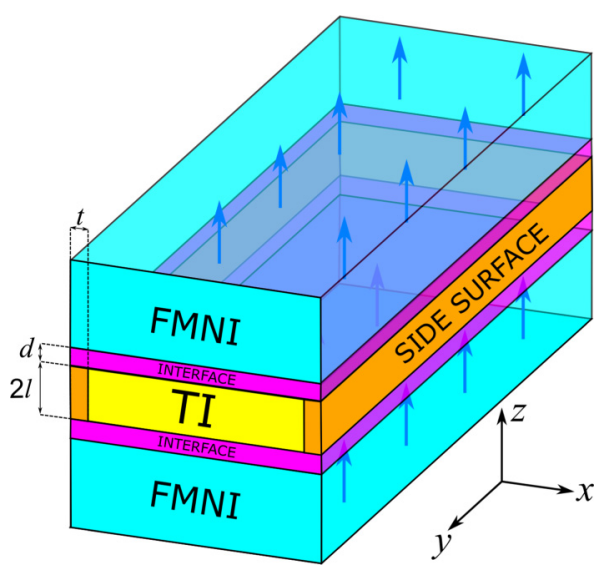

FIG. 1. Scheme of the FMNI/TI/FMNI trilayer. The TI film, indicated by yellow color, is sandwiched by the FMNI slabs, indicated by cyan color. The FM order in the FMNI slabs is symbolized by the set of the vertical blue arrows oriented along the $\mathbf{z}$ quantization axis, which is perpendicular to the interface plane. The interface potential $\mathbb{U}$ is localized at the TI/FMNI interfaces, which are highlighted in pink. The side surfaces of the film, highlighted in orange, host the edge potential $\mathbb{V}$. In the QAHE regime, electrons propagate along the side surfaces.

up/down, respectively. The model 3D Hamiltonian has a form of a power expansion with respect to the wave vector $\mathbf{k}$ around the $\Gamma$ point $(\mathbf{k}=0)$ of the Brillouin zone in the presence of time-reversal and space-inversion symmetries. For the sake of calculation simplicity, we apply the particle-hole symmetric and isotropic $4 \times 4$ Hamiltonian

$$
\mathbb{H}(\mathbf{k})=\Xi(\mathbf{k}) \tau_{z} \otimes \sigma_{0}+\mathrm{A} \tau_{x} \otimes(\boldsymbol{\sigma} \cdot \mathbf{k}),
$$

where $\Xi(\mathbf{k})=\Xi_{0}-\mathrm{B} k^{2}, 2 \Xi_{0}$ is a band gap, B describes a band curvature, $\sigma_{\alpha}$ and $\tau_{\alpha}(\alpha=0, x, y, z)$ denote the Pauli matrices in the spin and orbital spaces, respectively. The Hamiltonian (2) captures the remarkable feature of the band structure: the inverted order in energy of the basis states near $\mathbf{k}=0$, which arises under the condition $\Xi_{0} \mathrm{~B}>0$ due to strong SOC. The operator $\mathbb{H}(\mathbf{k})$ acts in the space of EFs of the Bloch states represented by 4 -spinors in the basis $u_{\Gamma}$.

The effective influence of the surrounding media on electrons, residing in the Bloch states $u_{\Gamma}$, is described by the confining potential $\mathbb{W}(\mathbf{r})$. We consider the structurally symmetric FMNI/TI/FMNI trilayer, in which both surfaces of the TI film are interfaced at $|z|=l$ with the FMNI material slabs. Besides, the TI film is bounded in the interface plane $(x, y)$ by the side surfaces meeting a topologically trivial medium at a circumference $\Upsilon(x, y)=0$. In such case, the confining potential in Eq. (1) can be represented as

$$
\mathbb{W}(\mathbf{r}) \approx d \mathbb{U} \delta(|z|-l)+t \mathbb{V} \delta[\Upsilon(x, y)],
$$

where $\delta(z)$ is the delta function. The potential $\mathbb{W}$ is assumed to be localized at both the TI/FMNI interfaces (on the scale $\sim d$ ) and the side surfaces (on the scale $\sim t$ ), as it is sketched in Fig. 1. In Eq. (3) for $\mathbb{W}$ we adopt the local approximation since EF varies very slowly as compared with the scales $\sim d$ and $\sim t$, which are of the order of a few interatomic distances. Following the concept of Refs. [37-39], the IP matrix $\mathbb{U}$, represented in the basis $u_{\Gamma}$, reflects such effects as charge density transfer across the interface, a surface relaxation due to a lattice mismatch between constituents as well as an interfacial overlapping of the TI wave functions with the FMNI ones. When the 3D TI film is placed in close proximity to a nonmagnetic NI material, IP is spin independent so that the IP magnitude can be approximated by a diagonal matrix $\mathbb{U}_{p}=\operatorname{diag}\left\{U_{1}, U_{2}, U_{1}, U_{2}\right\}$ [37-39]. In the present case, when the TI film is sandwiched by the FMNI slabs, the $p_{z}$ states in the TI film are intermixed with the $3 d$ or $4 f$ states on the FMNI side of the interface via direct or indirect exchange coupling to give rise to the magnetic proximity effect. Here, we do not concern about the mechanism behind the proximity effect; this matter had been thoroughly discussed in Refs. [23,40,41]. We treat the magnetic proximity effect at the TI/FMNI interface introducing in the IP an exchange term $\mathbb{U}_{e x}$ that is determined by the direction and magnitude of the FMNI magnetic moments. The latter are assumed to be out of plane and parallel aligned. Pointing the quantization axis normal to the interface, the exchange term can be parametrized by two real components as $\mathbb{U}_{e x}=\operatorname{diag}\{G, F,-G,-F\}$. These components mimic the exchange energies at the interface, $2 G$ and $2 F$, for the states $|+\sigma\rangle$ and $|-\sigma\rangle$, respectively. Generally speaking, the energies $G$ and $F$ are relatively small, $|G, F| \ll \Xi_{0}$. The total IP matrix is given by $\mathbb{U}=\mathbb{U}_{p}+\mathbb{U}_{e x}$. The microscopic details of the TI/FMNI interfaces in real heterostructures are unknown, nevertheless, varying the IP components $U_{1,2}, G, F$ in a wide energy range allows us to qualitatively model the proximity effect on the low-energy states. In the present continual approach, the effective edge potential at the side surfaces of the TI film is simulated by means of the diagonal matrix $\mathbb{V}=\operatorname{diag}\{V, V, V, V\}$.

\section{EFFECTIVE 2D HAMILTONIAN OF THE FMNI/TI/FMNI TRILAYER}

The TI film is assumed to be sandwiched by alike FMNI material slabs with the parallel directions of the magnetization. Postponing the analysis of the effects of the interface roughness and the side surface with the effective potential $\mathbb{V}$ until Sec. V, here we assume that the TI/FMNI interfaces at $z=l$ and $-l$ are perfectly flat and the TI film is infinite in the plane $(x, y)$. We adopt the variational procedure for the energy functional, which has been developed in Ref. [42], to formulate the boundary problem for EF at the interfaces. The corresponding equations in the compact form are

$$
\begin{gathered}
{\left[\mathbb{H}\left(\kappa,-i \partial_{z}\right)-\mathbb{I} E\right] \Theta(\kappa, z)=0,} \\
\left.i \frac{\delta \mathbb{H}\left(\boldsymbol{\kappa},-i \partial_{z}\right)}{\delta\left(-i \partial_{z}\right)} \Theta(\kappa, z)\right|_{|z|= \pm l}=\mp 2 d \mathbb{U}(\kappa) \Theta(\kappa, \pm l),
\end{gathered}
$$

where the in-plane wave vector $\boldsymbol{\kappa}=\left(k_{x}, k_{y}\right)$ is a good quantum number and $\partial_{z}=\partial / \partial z, \mathbb{I}$ is a unit $4 \times 4$ matrix. The eigenvalues of the boundary problem (4) and (5) have a form of the two-dimensional (2D) energy bands $E(\kappa)$, the eigenfunctions $\Theta(\kappa, z)$ are the smooth and continuous EFs inside the TI film at $|z|<l$. In our approach, the natural boundary conditions (5), imposed on EF at the TI/FMNI interfaces, involve the effective spin-dependent IP [39]. Note that the use of the open boundary condition, when EF has zero amplitude at the 
interfaces $\Theta( \pm l)=0$, does not permit modeling the magnetic proximity effect.

Equations (4) and (5) can be solved analytically at $\kappa=$ 0 when the Hamiltonian acquires a block-diagonal form $\mathbb{H}\left(0,-i \partial_{z}\right)=\operatorname{diag}\left\{\mathcal{H}^{\uparrow}\left(-i \partial_{z}\right), \mathcal{H}^{\downarrow}\left(-i \partial_{z}\right)\right\}$ with

$$
\mathcal{H}^{\sigma}\left(-i \partial_{z}\right)=\tau_{z}\left(B \partial_{z}^{2}+\Xi_{0}\right)+i \tau_{x} \sigma A \partial_{z},
$$

where the superscript $\sigma=\uparrow / \downarrow$ means up/down projection of electron spin onto the quantization axis. The lower block $\mathcal{H}^{\downarrow}$ is the time reversal of the upper block $\mathcal{H}^{\uparrow}$. The bispinor functions $\varphi^{\sigma}(z)$ and $\chi^{\sigma}(z)$, presenting the components of $\mathrm{EF}$ $\Theta(\kappa=0, z)$, are solutions of the boundary problem:

$$
\begin{aligned}
& \mathcal{H}^{\sigma}\left(-i \partial_{z}\right) \varphi^{\sigma}(z)=E_{\varphi}^{\sigma} \mathcal{I} \varphi^{\sigma}(z), \\
& \mathcal{H}^{\sigma}\left(-i \partial_{z}\right) \chi^{\sigma}(z)=E_{\chi}^{\sigma} \mathcal{I} \chi^{\sigma}(z), \\
& \left.\mathcal{G}^{\sigma}\left(-i \partial_{z}, z\right) \varphi^{\sigma}(z)\right|_{z= \pm l}=0, \\
& \left.\mathcal{G}^{\sigma}\left(-i \partial_{z}, z\right) \chi^{\sigma}(z)\right|_{z= \pm l}=0,
\end{aligned}
$$

where the operator $\mathcal{G}^{\sigma}$ is given by

$$
\begin{aligned}
\mathcal{G}^{\sigma}\left(-i \partial_{z}, z\right)= & -\tau_{0} \operatorname{sgn}(z) d\left(U_{1}^{\sigma}+U_{2}^{\sigma}\right)+\tau_{z}\left[2 B \partial_{z}\right. \\
& \left.-\operatorname{sgn}(z) d\left(U_{1}^{\sigma}-U_{2}^{\sigma}\right)\right]+i \tau_{x} \sigma A,
\end{aligned}
$$

$\mathcal{I}$ is a unit $2 \times 2$ matrix, $U_{1}^{\sigma}=U_{1}+\sigma G, U_{2}^{\sigma}=U_{2}+\sigma F$.

Due to the space symmetry of the system along the $\mathbf{z}$ axis, the trial solutions of Eqs. (7) and (8) can be represented as superpositions of the even functions $\cosh \left(q_{\varphi 1,2}^{\sigma} z\right)$ and $\cosh \left(q_{\chi 1,2}^{\sigma} z\right)$ for bound states or the odd functions $\sinh \left(q_{\varphi 1,2}^{\sigma} z\right)$ and $\sinh \left(q_{\chi 1,2}^{\sigma} z\right)$ for antibound states, where the superposition coefficients are functions of $l$ and $U_{1,2}^{\sigma}$. The momenta $q_{\varphi 1,2}^{\sigma}=q_{1,2}\left(E_{\varphi}^{\sigma}\right)$ and $q_{\chi 1,2}^{\sigma}=q_{1,2}\left(E_{\chi}^{\sigma}\right)$, specifying a scale of the EF spatial variation, are connected with the energy via the relation

$$
\left[q_{1,2}(E)\right]^{2}=-k_{m}^{2} \pm \sqrt{E^{2}-E_{m}^{2}} / B .
$$

Here, the following notations are used: $k_{m}^{2}=(1-2 \lambda) \Xi_{0} / B$, $E_{m}^{2}=4 \lambda(1-\lambda) \Xi_{0}^{2}$, and the parameter $\lambda=A^{2} / 4 B \Xi_{0}$ featuring the bulk band structure of 3D TI. In the case of $\lambda<1$, the bulk spectrum of the Hamiltonian (2) takes a "camelback" shape with a minimal gap of $2 E_{m}$ at nonzero momentum $k_{m}$.

The $\varphi$ state $\varphi_{n}^{\sigma}(z)$ and the $\chi$ state $\chi_{n}^{\sigma}(z)$ have opposite space parities, the index $n(n=0, \pm 1, \pm 2, \ldots)$ enumerates the discrete levels of the electron motion along the $\mathbf{z}$ axis in the film geometry due to the quantum size effect. As shown in Ref. [42], over a wide range of the model parameters, the low-energy states $(n=0)$ are clearly separated in energy from high-energy states $(|n| \geqslant 1)$, i.e., $\mid E_{\varphi, \chi 0}^{\sigma}-$ $E_{\varphi, \chi n}^{\sigma} \mid \gtrsim \Xi_{0} \sqrt{\lambda(1-\lambda)}$. This allows us (here, we skip the details which have been explained in Refs. [43,44]) to project the complete set of the eigenstates of the problem (7) and (8) onto a subspace spanned by a minimal orthogonal basis set composed of the four low-lying states at $\kappa=0, \varphi_{0}^{\sigma}(z)$, and $\chi_{0}^{\sigma}(z)$, and then construct the perturbation theory for the low-energy states using this truncated basis in terms of a small value of $\mathbb{H}\left(\kappa,-i \partial_{z}\right)-\mathbb{H}\left(0,-i \partial_{z}\right)$. By applying the scheme
[42-44], we derive the effective 2D Hamiltonian specific for the TI film in the FMNI/TI/FMNI trilayer:

$$
\begin{gathered}
\mathbb{H}_{\text {eff }}(\boldsymbol{\kappa})=\operatorname{diag}\left\{\mathcal{E}^{\Uparrow}(\kappa) \mathcal{I}+\mathcal{F}^{\Uparrow}(\kappa), \mathcal{E}^{\Downarrow}(\kappa) \mathcal{I}+\mathcal{F}^{\Downarrow}(\kappa)\right\}, \\
\mathcal{F}^{\Uparrow}(\kappa)=\left(\begin{array}{cc}
\Delta^{\Uparrow}(\kappa) & \mathcal{A} k_{-} \\
\mathcal{A}^{*} k_{+} & -\Delta^{\Uparrow}(\kappa)
\end{array}\right), \\
\mathcal{F}^{\Downarrow}(\kappa)=\left(\begin{array}{cc}
-\Delta^{\Downarrow}(\kappa) & -\mathcal{A}^{*} k_{-} \\
-\mathcal{A} k_{+} & \Delta^{\Downarrow}(\kappa)
\end{array}\right),
\end{gathered}
$$

$k_{ \pm}=k_{x} \pm i k_{y}$. The Hamiltonian (11) is split into two blocks defined by opposite projections of the pseudospin degree of freedom (symbolized by $\Sigma=\Uparrow / \Downarrow$ ), which is a good quantum number [44]. We use the notations

$$
\begin{gathered}
\mathcal{E}^{\Sigma}(\kappa)=\mathcal{E}_{0}^{\Sigma}-D^{\Sigma} \kappa^{2}, \\
2 \mathcal{E}_{0}^{\Sigma}=E_{\varphi 0}^{\sigma}+E_{\chi 0}^{-\sigma}, 2 D^{\Sigma}=B_{\varphi 0}^{\sigma}+B_{\chi 0}^{-\sigma}, \\
\Delta^{\Sigma}(\kappa)=\Delta_{0}^{\Sigma}-b^{\Sigma} \kappa^{2}, \\
2 \Delta_{0}^{\Sigma}=E_{\varphi 0}^{\sigma}-E_{\chi 0}^{-\sigma}, 2 b^{\Sigma}=B_{\varphi 0}^{\sigma}-B_{\chi 0}^{-\sigma} .
\end{gathered}
$$

It is implied that the permutation $\sigma \rightarrow-\sigma$ entails $\Sigma \rightarrow-\Sigma$ (for instance, $\sigma=\uparrow \rightarrow-\sigma=\downarrow$ entails $\Sigma=\Uparrow \rightarrow-\Sigma=\Downarrow$ ). The energies $E_{\varphi 0}^{\sigma}$ and $E_{\chi 0}^{\sigma}$ are defined above, the reciprocal masses are given by the matrix elements $B_{\varphi}^{\sigma}=B \int_{-l}^{l} d z\left(\varphi_{0}^{\sigma}\right)^{\dagger} \sigma_{z} \varphi_{0}^{\sigma}, B_{\chi}^{\sigma}=B \int_{-l}^{l} d z\left(\chi_{0}^{\sigma}\right)^{\dagger} \sigma_{z} \chi_{0}^{\sigma}$ under the normalization $\int_{-l}^{l} d z\left(\varphi_{0}^{\sigma}\right)^{\dagger} \sigma_{0} \varphi_{0}^{\sigma}=\int_{-l}^{l} d z\left(\chi_{0}^{\sigma}\right)^{\dagger} \sigma_{0} \chi_{0}^{\sigma}=1$. In turn, $\mathcal{A}=A_{\varphi \chi}^{\sigma,-\sigma}=A \int_{-l}^{l} d z\left(\varphi_{0}^{\sigma}\right)^{\dagger} \sigma_{x} \chi_{0}^{-\sigma}, A_{\chi \varphi}^{\sigma,-\sigma}=\left[A_{\varphi \chi}^{-\sigma, \sigma}\right]^{*}$, $A_{\chi \varphi}^{\sigma,-\sigma}=-A_{\chi \varphi}^{-\sigma, \sigma}$.

As long as we address an analytical description of electronic properties of the TI/FMNI heterostructure, the structurally symmetric FMNI/TI/FMNI trilayer is naturally regarded as a basic model unit. Since the structural inversion asymmetry is absent, the Rashba-type contribution to an energy band splitting is explicitly equal to zero, and the Hamiltonian (11) is block diagonal.

It is worth noting that in our approach the parameters $\mathcal{E}_{0}^{\Sigma}, D^{\Sigma}, \Delta_{0}^{\Sigma}, b^{\Sigma}, \mathcal{A}$ specifying the Hamiltonian $\mathbb{H}_{\text {eff }}$ depend not only on the characteristics of the 3D TI bulk spectrum $\Xi_{0}, B, A$, but also on the film thickness $2 l$ and the IP components $U_{1,2}^{\sigma}$. The corresponding analytical expressions are highly cumbersome. Therefore, we restrict our consideration to the case of either the strong IP, $\left|\left(U_{1}^{\sigma}\right)^{-1}+\left(U_{2}^{\sigma}\right)^{-1}\right| \Xi_{0} \ll 1$, or the weak IP, $\left|U_{1}^{\sigma}+U_{2}^{\sigma}\right| \Xi_{0}^{-1} \ll 1$, for the TI film with the thickness exceeding the EF variation scale. We describe the case with $\lambda<1$ and $\left|E_{\varphi 0}^{\sigma}\right|,\left|E_{\chi 0}^{\sigma}\right|<E_{m}$, when the momenta of Eq. (10) are complex, $q_{\varphi 1}^{\sigma}=\left(q_{\varphi 2}^{\sigma}\right)^{*}, q_{\chi 1}^{\sigma}=\left(q_{\chi 2}^{\sigma}\right)^{*}$.

In the case of the strong IP, one can obtain the following estimations:

$$
\begin{gathered}
\mathcal{E}_{0}^{\Sigma}=-\frac{|A| \Xi_{0}}{d}\left(\frac{U_{1}}{U_{1}^{2}-G^{2}}+\frac{U_{2}}{U_{2}^{2}-F^{2}}\right), \quad D^{\Sigma}=\frac{B}{\Xi_{0}} \mathcal{E}_{0}^{\Sigma}, \\
\Delta_{0}^{\Sigma}=\Delta_{0}+\operatorname{sgn}(\Sigma) \omega
\end{gathered}
$$




$$
\begin{gathered}
\Delta_{0}=-\frac{2|A| w_{0}}{1-\lambda} \sin \left(2 w_{0} l\right) \exp \left(-2 q_{0} l\right), \\
\omega=-\frac{|A| \Xi_{0}}{d}\left(\frac{G}{U_{1}^{2}-G^{2}}+\frac{F}{U_{2}^{2}-F^{2}}\right) \\
b^{\Sigma}=\beta-\operatorname{sgn}(\Sigma) \frac{B}{\Xi_{0}} \omega \\
\beta=\frac{2|A|}{1-\lambda} l \cos \left(2 w_{0} l\right) \exp \left(-2 q_{0} l\right) .
\end{gathered}
$$

Here and following, $\operatorname{sgn}(\Sigma)=+/-$ refers to the pseudospin projection $\Uparrow / \Downarrow$, respectively.

In the case of the weak IP, one gets the explicit expressions

$$
\begin{gathered}
\mathcal{E}_{0}^{\Sigma}=\frac{2 q_{0} d}{1+\lambda}\left(U_{1}+U_{2}\right), D^{\Sigma}=0, \\
\Delta_{0}^{\Sigma}=\Delta_{0}+\operatorname{sgn}(\Sigma) \omega, \\
\Delta_{0}=\frac{2|A| w_{0}}{1+\lambda} \sin \left(2 w_{0} l\right) \exp \left(-2 q_{0} l\right), \\
\omega=\frac{2 q_{0} d}{1+\lambda}(G+F), \\
b^{\Sigma}=\beta=-\frac{2|A|}{1+\lambda} l \cos \left(2 w_{0} l\right) \exp \left(-2 q_{0} l\right) .
\end{gathered}
$$

Equations (17)-(27) for the components of the Hamiltonian (11) as functions of the TI thickness, the IP matrix elements, and the parameters of the 3D TI material band structure are derived under two conditions: (1) assuming the leading order in $\left|U_{1,2}^{\sigma}\right|^{-1} \Xi_{0}$ or $\left|U_{1,2}^{\sigma}\right| \Xi_{0}^{-1}$; (2) the overlap between the states arising at the opposite interfaces $\exp \left[-2 \operatorname{Re}\left(q_{\varphi 1,2}^{\sigma}\right) l\right]$ and $\exp \left[-2 \operatorname{Re}\left(q_{\chi 1,2}^{\sigma}\right) l\right]$ is small. In such a limit, one has $\operatorname{Re}\left(q_{\varphi 1,2}^{\sigma}\right)=\operatorname{Re}\left(q_{\chi 1,2}^{\sigma}\right)=q_{0}=|A| / 2 B$ and $\operatorname{Im}\left(q_{\varphi 1,2}^{\sigma}\right)=\operatorname{Im}\left(q_{\chi 1,2}^{\sigma}\right)=w_{0}=q_{0} \sqrt{\lambda^{-1}-1}$. The term $\mathcal{E}^{\Sigma}(\boldsymbol{\kappa})$ (13) accounts for the particle-hole asymmetry caused by IP [see Eqs. (17) and (23)]. The diagonal components in $\mathcal{F}^{\Sigma}(\boldsymbol{\kappa})$ (12) stem, on the one hand, from the overlap of the states and, on the other hand, from the spin-dependent scattering at the interfaces. For example, the term $\Delta_{0}^{\Sigma}$ of Eq. (18) or (24) is a sum of the proximity-induced exchange splitting $\omega$ and the hybridization $\Delta_{0}$. The former is not only proportional to the magnetic moment in an adjoining FMNI, but is also controlled by the IP spin-independent components, as is described, e.g., by Eq. (20). The latter, according to Eqs. (19) and (25), is mostly influenced by the TI thickness. Besides, both $\Delta_{0}$ and $\omega$ depend on the band structure parameters of the $3 \mathrm{D}$ TI material. The dispersion parameter $b^{\Sigma}$ also contains the hybridization contribution $\beta$ [Eqs. (22) and (27)] caused by the overlap of the states coming from the opposite interfaces, and the proximity-induced exchange term $\sim \omega$ [Eqs. (20) and (26)]. If the IP is either weak or strong, the magnitude of the off-diagonal terms in Eq. (12) does not depend on $l$ and can be estimated as $|\mathcal{A}|=2 \sqrt{\lambda \Xi_{0} B}$.

In our approach, the interface states, which are spin polarized by FMNI, penetrate the TI film over the length of the order of $\left(2 q_{0}\right)^{-1}$. As a result, on this scale, the near-interface regions in the film acquire a short-range out-of-plane magnetic order [41]. As demonstrated in Refs. [40,41], an essential feature of the TI/FMNI heterostructure is that the magnetic proximity effect is accompanied by significant charge transfer across the interface which entails a band bending. Here, this important electrostatic phenomenon is included in the microscopic model through the spin-independent part of IP, $\mathbb{U}_{p}$, taken in the local approximation. The spatial behavior of the low-energy states near the interfaces is directly regulated by the strength of the potential $\mathbb{U}_{p}$ [42]. Significantly, in the case of the strong IP, the EF vanishes at the interface as $\left|\varphi_{0}^{\sigma}( \pm l)\right|,\left|\chi_{0}^{\sigma}( \pm l)\right| \sim\left|U_{1}^{-1}+U_{2}^{-1}\right| \ll \Xi_{0}^{-1}$. For that reason, as it follows from Eq. (20), the induced exchange splitting is drastically suppressed by the potential scattering at the interface $\omega \sim\left(\frac{G}{U_{1}^{2}}+\frac{F}{U_{2}^{2}}\right)$. In turn, in the weak IP limit, the EF magnitude reaches a maximal value at the interface $\omega \sim$ $(G+F)$ [see Eq. (26)].

The spectrum of the Hamiltonian (11) consists of four branches expressed as

$$
E_{ \pm}^{\Sigma}(\kappa)=\mathcal{E}^{\Sigma}(\kappa) \pm \sqrt{\left[\Delta^{\Sigma}(\kappa)\right]^{2}+|\mathcal{A}|^{2} \kappa^{2}}
$$

When $D^{2}<b^{2}$, the energy gap between the electronlike band $E_{+}^{\Sigma}(\kappa)$ and the holelike band $E_{-}^{\Sigma}(\kappa)$ appears. The difference $E_{+}^{\Sigma}-E_{-}^{\Sigma}$ at $\kappa=0$ is equal to $2\left|\Delta_{0}^{\Sigma}\right|$.

\section{QUANTIZED HALL RESPONSE AND TOPOLOGICAL PHASE DIAGRAM OF THE FMNI/TI/FMNI TRILAYER}

The low-energy physics of the TI thin film sandwiched by the FMNI slabs is controlled by the low-energy spin-polarized electron states $\varphi_{0}^{\sigma}(z)$ and $\chi_{0}^{\sigma}(z)$, arising inside the 3D TI bulk gap. These states are very sensitive to the material and size characteristics of the trilayer. In Eqs. (18)-(22) and (24)-(27), this fact is explicitly represented by the dependence of the diagonal terms $\Delta^{\Sigma}(\kappa)$, on the thickness and the IP components, as well as on the 3D TI band structure. According to the Chern insulator concept [8], the terms $\Delta^{\Sigma}(\kappa)$ control whether the system is in a topological phase or not. It means that the heterostructure approach allows us to tune the topological order in a controllable way, simply by changing the aforesaid characteristics.

It is possible to assign the intrinsic Hall response $\sigma_{x y}^{\Sigma}$ to each block in Eq. (12). It is convenient to represent the response through the Chern number $C^{\Sigma}[8]$ as $\sigma_{x y}^{\Sigma}=\frac{e^{2}}{h} C^{\Sigma}(h$ is the Planck constant, $e$ is the electron charge). The Chern number is the integral of the Berry curvature over the first Brillouin zone [8]. When the Hamiltonian is in the form (12), the Berry curvature is obtained as [44]

$$
\Omega^{\Sigma}(\kappa)=\operatorname{sgn}(\Sigma) \frac{|\mathcal{A}|^{2}\left(\Delta_{0}^{\Sigma}+b^{\Sigma} \kappa^{2}\right)}{2\left\{\left[\Delta^{\Sigma}(\kappa)\right]^{2}+|\mathcal{A}|^{2} \kappa^{2}\right\}^{\frac{3}{2}}} .
$$

Since the function $\Omega^{\Sigma}(\kappa)$ decays quickly enough with increasing $\kappa$, the integral over the 2D Brillouin zone can be replaced by the integral over the infinite plane $\left(k_{x}, k_{y}\right)$. This leads to the integer-valued factor $C^{\Sigma}=\frac{1}{2} \operatorname{sgn}(\Sigma)\left[\operatorname{sgn}\left(b^{\Sigma}\right)+\right.$ $\left.\operatorname{sgn}\left(\Delta_{0}^{\Sigma}\right)\right]$, provided that the chemical potential $\mu$ lies within the gap, so that $|\mu|<2\left|\Delta_{0}^{\Sigma}\right| \sqrt{\Lambda^{\Sigma}\left(1-\Lambda^{\Sigma}\right)}$ at $0<\Lambda^{\Sigma}<\frac{1}{2}$ and otherwise $|\mu|<\left|\Delta_{0}^{\Sigma}\right|$, where $\Lambda^{\Sigma}=\frac{|\mathcal{A}|^{2}}{4 b^{\Sigma} \Delta_{0}^{\Sigma}}$. In the other case, the Chern number is no longer integer. Thus, in a trivial 
phase $C^{\Sigma}$ is zero and the Hall response vanishes, while in a topological phase it is an integer number \pm 1 and the response as a function of $\mu$ gets plateau with value of $\frac{e^{2}}{h}$.

One can define two topological invariants: the spin Chern number $C^{S}=\frac{1}{2}\left(C^{\Uparrow}-C^{\Downarrow}\right)$ and the total or charge Chern number $C^{C}=\frac{1}{2}\left(C^{\Uparrow}+C^{\Downarrow}\right)$, which identify the spin Hall effect and charge Hall effect, respectively. Since the time-reversal symmetry of the blocks is broken, the number $C^{C}$ gets a nontrivial value, which can lead to the appearance of QAHE. Under the condition that the chemical potential $\mu$ lies inside the energy gap in the spectrum (28), the quantized Hall response of the system is specified by a combination of the signs of $\Delta_{0}^{\Sigma}$ and $b^{\Sigma}$, namely, QSHE and QAHE are indexed by quantities $C^{S}=\frac{1}{4}\left[\operatorname{sgn}\left(\Delta_{0}^{\Uparrow}\right)+\operatorname{sgn}\left(\Delta_{0}^{\Downarrow}\right)+\operatorname{sgn}\left(b^{\Uparrow}\right)+\operatorname{sgn}\left(b^{\Downarrow}\right)\right]$ and $C^{C}=\frac{1}{4}\left[\operatorname{sgn}\left(\Delta_{0}^{\Uparrow}\right)-\operatorname{sgn}\left(\Delta_{0}^{\Downarrow}\right)+\operatorname{sgn}\left(b^{\Uparrow}\right)-\operatorname{sgn}\left(b^{\Downarrow}\right)\right]$, respectively. The topological phase transitions occur when the signs of $\Delta_{0}^{\Sigma}$ and $b^{\Sigma}$ change.

The principle behind the QAHE in the TI film with FM order is a competition between the hybridization gap and the exchange splitting energy $[33,34,45,46]$. As soon as the net magnetization appears in the TI film sandwiched between the FMNI slabs, the induced spin splitting of the topologically relevant states may withdraw the band inversion for one spin projection, while remaining the band inversion for the opposite spin projection, thus meeting the essential condition for the QAHE [33,34,45,46]. Both the hybridization of the interface states and the induced exchange splitting in the TI film give rise to the diagonal terms $\Delta^{\Sigma}(\kappa)$. And so, when we construct the quantum phase diagram of the FMNI/TI/FMNI trilayer, we ought to know the equations of boundaries between topologically distinct insulating phases determined by conditions $\Delta_{0}^{\Sigma}(l, \omega)=0$ and $b^{\Sigma}(l, \omega)=0$, expressed in terms of the corresponding characteristics of the system. For example, in the case of the strong potential $\mathbb{U}_{p}$, one can use Eqs. (18)-(22). For such trilayer the topological phase diagram is shown in Fig. 2, where the chemical potential is assumed to reside within both the gaps, related to the upper block $\mathcal{F} \Uparrow$ and the lower one $\mathcal{F}^{\Downarrow}$. One can see that topological phase transitions can be controlled by tuning the exchange field together with the film thickness. In the phase diagram, apart from the trivial phase areas (region 7) with $C^{S}=C^{C}=$ 0 , there are several regions with different nontrivial Chern numbers: the QSHE phase with $C^{S}=-1, C^{C}=0$ (region 2), and $C^{S}=1, C^{C}=0$ (region 5); the mixed QAHE+QSHE phase with $C^{S}=\frac{1}{2}, C^{C}=\frac{1}{2}$ (region 1 ), $C^{S}=-\frac{1}{2}, C^{C}=\frac{1}{2}$ (region 6), $C^{S}=-\frac{1}{2}, C^{C}=-\frac{1}{2}$ (region 3 ), and $C^{S}=\frac{1}{2}, C^{C}=$ $-\frac{1}{2}$ (region 4). The responses $\sigma_{x y}^{S}$ and $\sigma_{x y}^{C}$ remain unchanged and quantized according to the value and sign of the Chern number inside the corresponding phase and change abruptly at the phase boundary. The realization of the QAHE regime is naturally associated with the mixed QAHE+QSHE phase. Note that when the exchange field reverses from $\mathbf{z}$ to $-\mathbf{z}$, i.e., $F \rightarrow-F, G \rightarrow-G$, the anomalous Hall response changes its sign, $C^{C} \rightarrow-C^{C}$, while the spin Hall response does not alter, $C^{S} \rightarrow C^{S}$. Importantly, the trilayer can pass into the mixed QAHE+QSHE phase from the trivial phase or the QSHE phase, by varying both the film thickness and the exchange potential. Note, at finite value of the exchange splitting, there is no boundary between the trivial phase and

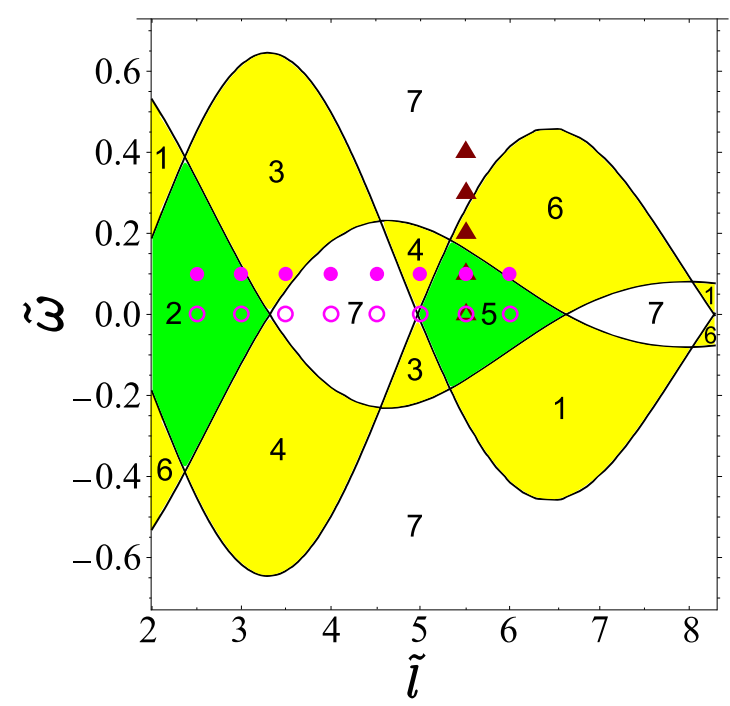

FIG. 2. Topological phase diagram of the FMNI/TI/FMNI trilayer in terms of the proximity-induced exchange splitting $\omega$ and the TI film thickness $l$ under strong potential $\mathbb{U}_{p}$ when $\lambda=0.1$. Here, $\widetilde{l}=l \sqrt{\frac{\Xi_{0}}{B}}, \widetilde{\omega}=\frac{\omega \sqrt{B}}{|| A \mid \sqrt{\Xi_{0}}}$, where $\omega$ is given by Eq. (20). One can observe three topologically distinct insulating phases characterized by the quantized spin Chern number and quantized charge Chern number and represented by the corresponding regions. White regions mark the trivial phase with $C^{S}=C^{C}=0$. Green regions mark QSHE phase with $\left|C^{S}\right|=1, C^{C}=0$. Yellow regions mark the mixed QAHE+QSHE phase with $\left|C^{S}\right|=\frac{1}{2},\left|C^{C}\right|=\frac{1}{2}$. Boundaries selecting different phases are given by equations $\Delta_{0}^{\Sigma}(l, \omega)=0$ and $b^{\Sigma}(l, \omega)=$ 0 . The triangles and circles (hollow and solid) mark the points in the parameter space, at which we below compute the electron spectrum and the Berry curvature.

the QSHE phase. The phase diagram for the case of $\lambda>1$ had been calculated in Ref. [47].

In the case of the weak IP, when the EF amplitude at the interface attains maximum, the topological phase diagram is in general similar to that shown in Fig. 2, albeit some features are modified. One can describe the dependence of the spin and charge Chern numbers on the thickness and the exchange splitting, taking into account Eqs. (23)-(27). Comparing Eqs. (25) and (27) with Eqs. (19) and (22) we infer that, when the potential $\mathbb{U}_{p}$ changes from strong to weak limit, the dependencies of $C^{S}$ and $C^{C}$ on $l$ remain oscillating with the period $\frac{\pi}{w_{0}}$, but the oscillation phase shifts by the half-period. It is not surprising that the behavior of the Chern numbers does not change in principle in the opposite cases of the strong or weak IP. Indeed, the topological properties of the system are determined by the electron band structure of the material. In the FMNI/TI/FMNI trilayer, the boundary condition at the interface may only affect some details of the dependencies $C^{S}(l)$ and $C^{C}(l)$. Put simply, the IP is responsible for the phase shift of the oscillation of the spin and charge Chern numbers with the film thickness. The IP can also influence the oscillation period.

To reflect the effect of the TI film thickness and the interface exchange coupling on electron characteristics of the trilayer, we chose points on the diagram plane in Fig. 2 in two directions: horizontal (increasing $\tilde{l}$ at fixed $\tilde{\omega}=0 / \tilde{\omega}=0.1$ 
depicted by hollow/solid pink circles) and vertical (varying the magnitude of $\tilde{\omega}$ at fixed $\tilde{l}=5.5$ depicted by brown triangles). For these points we show evolution of the band dispersion and Berry curvature with increase of the thickness in Fig. 3 and the exchange splitting magnitude in Fig. 4. These figures illustrate clearly how the relative arrangement of the band branches changes, when the system moves from one phase to another. On the upper row of plots, blue and red dispersion branches are obtained for the upper block $\mathcal{F}^{\Uparrow}$, cyan and orange ones are obtained for the lower block $\mathcal{F}^{\Downarrow}$. Background color shows the phase that the system is turned into, according to Fig. 2. Thus, the normal band progression is presented by sequence of blue/cyan bands as the valence bands and red/orange as conduction bands. In the QSHE phase, both pairs of the bands are inverted. In Fig. 3 one can see that in the mixed QAHE+QSHE phase, which exists at $\widetilde{l}=3.5,5.0,6.0$ (the plots with the yellow background on the right side), a pair of the bands for one block is inverted, while a pair of the bands for another block is in normal order. Similar alignment of the bands is shown in Fig. 4 for the mixed QAHE+QSHE phase existing at $\widetilde{\omega}=0.2$. At $\tilde{\omega}=0$, bands are degenerate due to $\Delta_{0}^{\Uparrow}=\Delta_{0}^{\Downarrow}$ and $b^{\Uparrow}=b^{\Downarrow}$. In the case of very thin TI film, for example at $\tilde{l}=2.5$, the spectrum is of "camelback" shape around $k=0$.

As far as Berry curvature is concerned, it is very sensitive to changes of the parameters $\Delta_{0}^{\Sigma}$ and $b^{\Sigma}$, when the trilayer passes from one phase to another. In the lower rows of Figs. 3 and 4 , the black/pink curve describes $\Omega^{\Uparrow}(\kappa) / \Omega^{\Downarrow}(\kappa)$. At $\tilde{\omega}=$ 0 , Berry curvatures are symmetric respective to energy axis, while at finite $\tilde{\omega}$ they become asymmetric. The details of the Berry curvature distribution vary remarkably depending on whether the parameters $\Delta_{0}^{\Sigma}$ and $b^{\Sigma}$ have the same or different signs.

\section{HALL CONDUCTIVITY OF THE FMNI/TI/FMNI TRILAYER WITH ROUGH INTERFACES}

A natural and greatly important topic for the heterostructure design is how the transport properties are affected by the interface inhomogeneities. In fabricated samples, the interfaces between constituents are not perfect due to an unavoidable roughness, atomic interdiffusion, amorphization, lattice mismatch, etc. The atomic force and scanning tunneling microscopy topographic images of thin films of tetradymite semiconductors show varying morphology and crystalline quality depending on fabrication method and substrate material on which they are grown $[16,48,49]$. For example, the surface of $\mathrm{V}$-doped $(\mathrm{Bi}, \mathrm{Sb})_{2} \mathrm{Te}_{3}$ film grown on $\operatorname{InP}(111)$ by molecular beam epitaxy exhibits the triangular Te-terminated terraces of width $\mathcal{L}$ up to $140 \mathrm{~nm}$ separated by steps with height of $\approx 1 \mathrm{~nm}$, while in the case of the $\mathrm{Si}(111)$ substrate, the surface looks like islands of $\mathcal{L} \lesssim 100 \mathrm{~nm}$ and height up to $12 \mathrm{~nm}$ with deep trenches between them [16].

The assumption on the perfect, i.e., atomically abrupt, TI/FMNI interfaces used heretofore is idealized, and transport measurements of heterostructures with rough interfaces could be more adequately explained on the base of the scenario, where the TI film thickness depends on the in-plane coordinate $l(x, y)$. Relying on calculations [50], where the kp model is parametrized using first-principles data for the tetradymite
TI materials, we adopt the following estimation for the band structure parameters of the simple Hamiltonian of Eq. (2): $|A|=0.2 \mathrm{eV} \mathrm{nm}, B=0.5 \mathrm{eV} \mathrm{nm}^{2}, \Xi_{0}=0.2 \mathrm{eV}$. These result in the values $\lambda=0.1, q_{0}=0.2 \mathrm{~nm}^{-1}$, and $w_{0}=0.6 \mathrm{~nm}^{-1}$, so that the hybridization gap oscillates with the period of $\sim_{w_{0}}^{\pi}=5.2 \mathrm{~nm}$. Hence, at the small or moderate value of the exchange splitting (e.g., $\widetilde{\omega} \simeq 0.1$ as it is shown in Fig. 2 ), the characteristic interval between neighboring phase boundaries is $L_{0} \lesssim \frac{\pi}{4 w_{0}}=1.3 \mathrm{~nm}$, which is comparable with the $\mathrm{QL}$ height of $h_{\mathrm{QL}} \approx 0.9 \mathrm{~nm}$ in the binary tetradymite TIs. The similar estimations of the oscillation period of the hybridization gap for the films of the TIs $\mathrm{Bi}_{2} \mathrm{Se}_{3}$ and $\mathrm{Bi}_{2} \mathrm{Te}_{3}$ have been obtained in the theoretical works $[44,51,52]$. These facts mean that the quantized conductivity regime can be highly sensitive to variation of the TI film thickness.

We assume that the roughness is sufficiently weak and smooth so that a mean distance between neighboring QL steps exceeds well a scale of the EF variation in the interface plane. Then, the results of the above study can be applied locally. In other words, the formation of different phases in the trilayer corresponds to local thickness of the TI film, and one can introduce the position-dependent Chern number $C^{\Sigma}(x, y)$ in the quasiclassical language. As long as the film thickness is a function of the in-plane coordinate, each domain $i$ is featured by its own pair of numbers $\left\{C_{i}^{\Uparrow}(x, y), C_{i}^{\Downarrow}(x, y)\right\}$. Therefore, relying on the phase diagram in Fig. 2, one can suggest that, due to unavoidable roughness, the TI thin film is broken down into topologically distinct domains separated from each other by domain walls spatially coincided with QL steps. Note that not every QL step is a domain wall. So, in the general case, by varying the TI film thickness while keeping a moderate value of the exchange field, one can distinguish seven phase domains with different numbers $\left\{C^{\Uparrow}(x, y), C^{\Downarrow}(x, y)\right\}$, summarized in Fig. 2. The domain regions are insulating, but the topologically protected 1D conducting channels occur along the domain walls separating the corresponding phases. A domain wall originated from a QL step can be referred to as an "inner edge" in contrast to an "outer edge" associated with the side surface that confines the TI film along its perimeter $\Upsilon(x, y)=0$ in the $(x, y)$ plane.

At the side surface, where the TI film is contiguous with trivial surroundings, there arise the outer edge states that are expected to support the quantized Hall conductance. Let the film interior phase be specified by the pair of the Chern numbers $\left\{C_{i}^{\Uparrow}, C_{i}^{\Downarrow}\right\}$. Then, the asymptotic decreasing of the outer edge state in the direction normal to the side surface $\Upsilon(x, y)=y$ is determined by the characteristic momentum $p_{i}^{\Sigma}$ :

$$
\begin{gathered}
\vartheta_{i}^{\Sigma}(x, y) \sim \exp \left(i k_{y} y\right) \exp \left(-p_{i}^{\Sigma} x\right), \\
p_{i}^{\Sigma}=\frac{\operatorname{sgn}\left(\Delta_{0 i}^{\Sigma} b_{i}^{\Sigma}\right)}{2\left|b_{i}^{\Sigma}\right|}\left[|\mathcal{A}|-\sqrt{\mathcal{A}^{2}-4 \Delta_{0 i}^{\Sigma} b_{i}^{\Sigma}}\right],
\end{gathered}
$$

where the parameters $\Delta_{0 i}^{\Sigma}$ and $b_{i}^{\Sigma}$ are associated with the phase $i$. On the other hand, close to the side surface $x<\left(p_{i}^{\Sigma}\right)^{-1}$, the behavior of the $\mathrm{EF} \vartheta_{i}^{\Sigma}(x, y)$ is strongly dominated by the effective outer edge potential (OEP) $\mathbb{V}$ [42]. The spin-resolved outer edge states possess the linear spectrum inside the 2D gap 

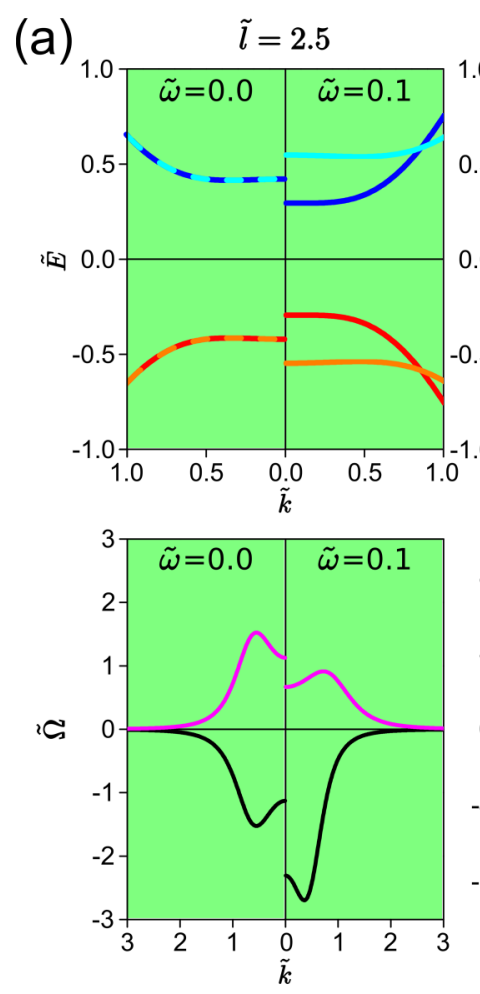

(b)
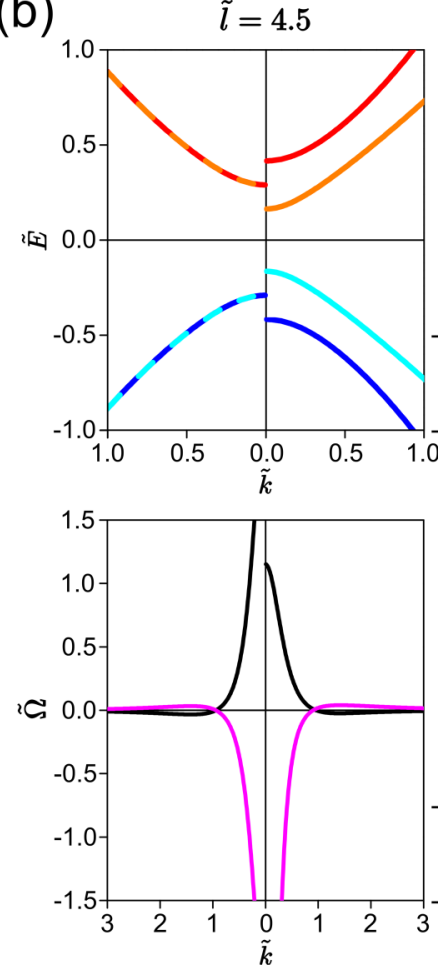
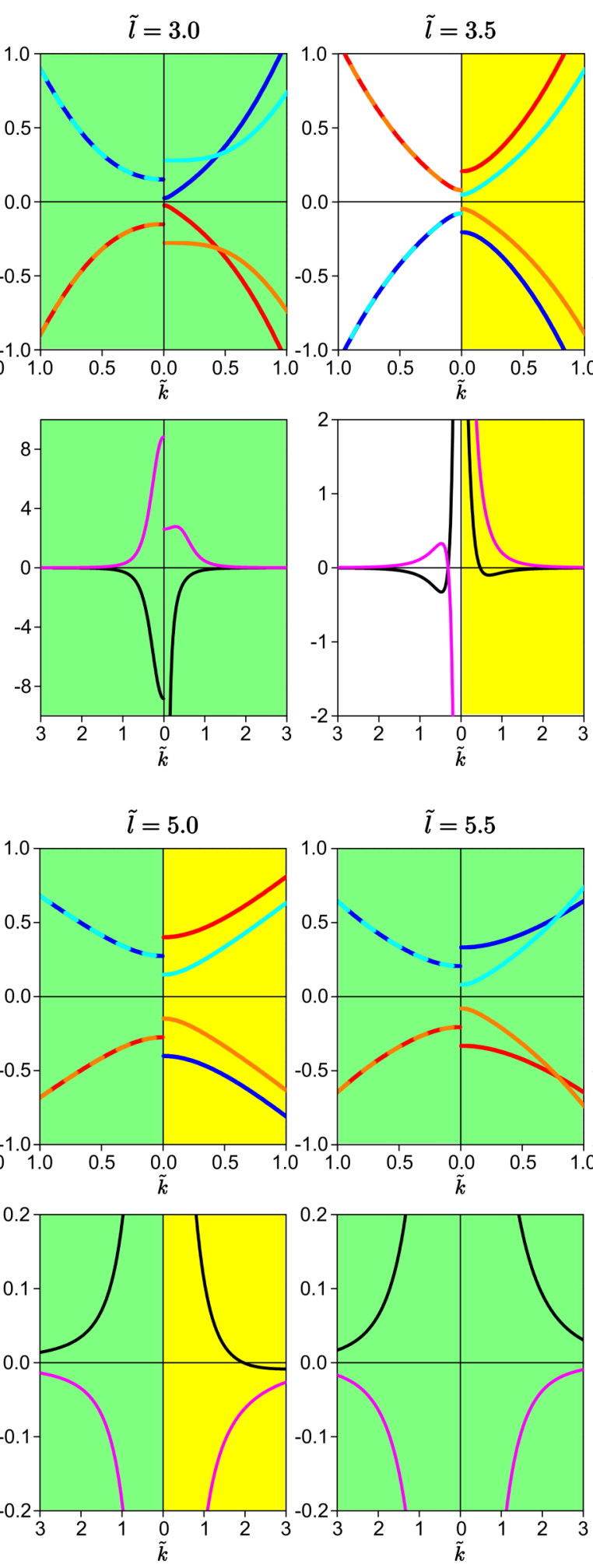
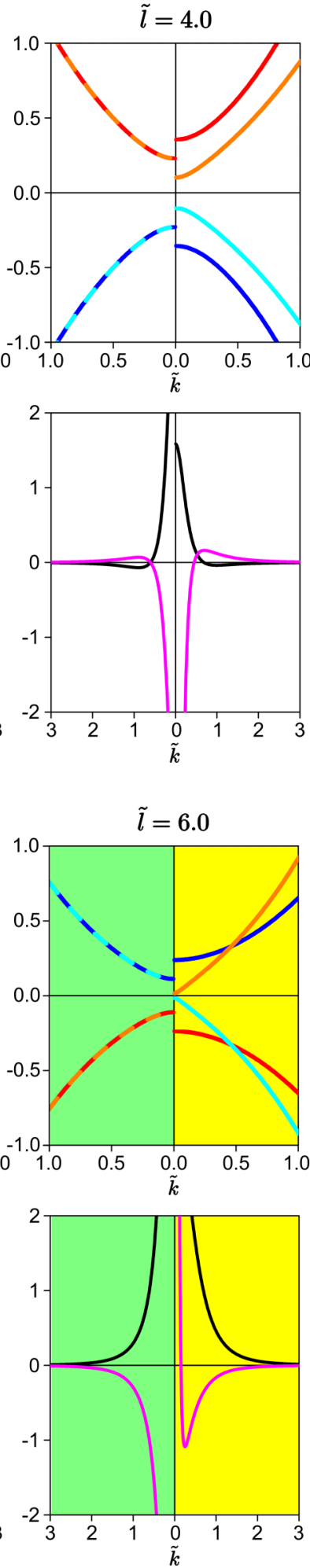

FIG. 3. Evolution of the dispersion relation $E_{ \pm}^{\Sigma}(\kappa)$ (upper row) and the Berry curvature $\Omega^{\Sigma}(\kappa)$ (lower row) with the TI film thickness [(a) for $\tilde{l}=2.5 \ldots 4.0$ and (b) for $\tilde{l}=4.5 \ldots 6.0]$ taking place in the FMNI/TI/FMNI trilayer. Here, $\widetilde{E}=\frac{E}{\Xi_{0}}, \widetilde{\Omega}=\Omega \frac{\Xi_{0}}{B}, \widetilde{k}=k_{x, y} \sqrt{\frac{B}{\Xi_{0}}}, \tilde{l}=l \sqrt{\frac{\Xi_{0}}{B}}$. In order to provide a comprehensive picture of the evolution, we choose eight different thicknesses denoted by solid circles at the finite value of $\widetilde{\omega}$ and by hollow circles in absence of the exchange splitting in the phase diagram in Fig. 2. The left part of every plot relates to $\widetilde{\omega}=0.0$, while the right part relates to $\widetilde{\omega}=0.1$. The dispersion of the four lowest subbands near the point $\kappa=0, E_{ \pm}^{\Sigma}(\kappa)$ and the momentum distribution of the Berry curvature for opposite quasispin polarizations $\Omega^{\Sigma}(\kappa)$ are drawn in the corresponding plots on the background with color associated with a quantum phase in the diagram in Fig. 2. The curves are computed taking the same parameters as were used in Fig. 2. 

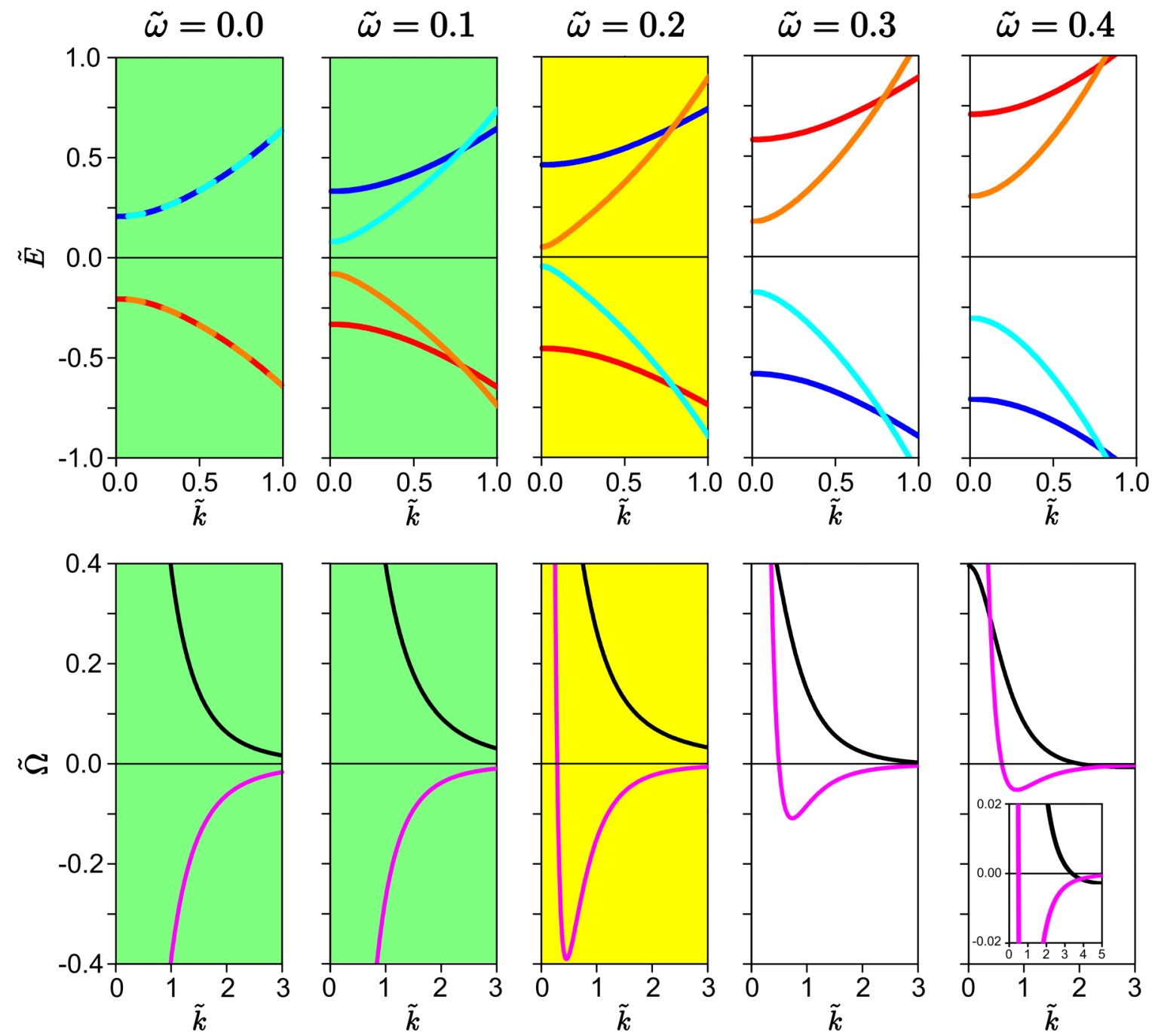

FIG. 4. Evolution of the dispersion relation $E_{ \pm}^{\Sigma}(\kappa)$ (upper row) and the Berry curvature $\Omega^{\Sigma}(\kappa)$ (lower row) with the exchange splitting taking place in the FMNI/TI/FMNI trilayer. Here, $\widetilde{E}=\frac{E}{\Xi_{0}}, \widetilde{\Omega}=\Omega \frac{\Xi_{0}}{B}, \widetilde{k}=k_{x, y} \sqrt{\frac{B}{\Xi_{0}}}, \widetilde{\omega}=\frac{\omega \sqrt{B}}{2|A| \sqrt{\Xi_{0}}}$. In order to provide a comprehensive picture of the evolution, we choose five different values of the exchange splitting denoted by triangles at the fixed thickness $\tilde{l}=5.5$ in the phase diagram in Fig. 2. The dispersion of the lowest four subbands near the point $\kappa=0, E_{ \pm}^{\Sigma}(\kappa)$ and the momentum distribution of the Berry curvature for opposite quasispin polarizations $\Omega^{\Sigma}(\kappa)$ are drawn in the corresponding plots on the background with color associated with a quantum phase in the diagram in Fig. 2. The curves are computed taking the same parameters as were used in Fig. 2.

$2 \Delta_{0 i}^{\Sigma}$, which is given to the first order in $k_{y}$ by

$$
\varepsilon^{\Sigma}\left(k_{y}\right)=\varepsilon_{0}^{\Sigma}(V)+\operatorname{sgn}(\Sigma)|\mathcal{A}| k_{y},
$$

where $\operatorname{sgn}(\Sigma)$ relates to the pseudospin index as $\operatorname{sgn}(\Sigma=\Uparrow$ $/ \Downarrow)=+1 /-1$. The OEP with strength $V$ gives rise to the Dirac point shift $\varepsilon_{0}^{\Sigma}(V)$ with respect to the middle of the gap in Eq. (28). The dependence $\varepsilon_{0}^{\Sigma}(V)$ has been described in [42]. In the case of strong or weak OEP, the shift is small $\varepsilon_{0}^{\Sigma}(V) \sim V^{-1}$ or, respectively, $\varepsilon_{0}^{\Sigma}(V) \sim V$, while in the case of moderate OEP, the Dirac point can merge into the 2D band continuum [42]. Besides, if the film interior is in the trivial phase with $\Delta_{0 i}^{\Sigma} b_{i}^{\Sigma}<0$, OEP of finite strength can create the outer edge states of the Rashba type [42], which do not traverse the 2D gap and, hence, are not topologically robust.

The inner edges host the low-energy helical or chiral electron states confined to the domain walls, across which the diagonal terms in Eq. (12) being the functions of the TI film thickness, $\Delta_{0}^{\Sigma}[l(x, y)]$ and $b^{\Sigma}[l(x, y)]$, change signs. Such a topologically protected state, interposed between regions of the different phases $i$ and $j$, is featured by the corresponding components $\left\{\Delta_{0 i}^{\Sigma}, b_{i}^{\Sigma}\right\}$ and $\left\{\Delta_{0 j}^{\Sigma}, b_{j}^{\Sigma}\right\}$. Generally speaking, there exist 21 different bound inner edge states, which correspond to a number of possible pair combinations of the phases specified by the Chern numbers $\left\{C_{i}^{\Uparrow}, C_{i}^{\Downarrow}\right\}$ and $\left\{C_{j}^{\Uparrow}, C_{j}^{\Downarrow}\right\}$. The key features of the energy spectrum and the EF asymptotic behavior of the inner edge state $\eta_{i j}^{\Sigma}(x, y)$ can be presented in a general form. If, for instance, the interdomain boundary $y=y_{0}$ is running along the $x$ axis, the decay of EF with the distance $\left|y-y_{0}\right|$ from the boundary is approximated by the expression

$$
\begin{aligned}
& \eta_{i j}^{\Sigma}(x, y) \sim \exp \left(i k_{x} x\right)\left\{h\left(y_{0}-y\right) \exp \left[p_{j}^{\Sigma}\left(y-y_{0}\right)\right]\right. \\
& \left.\quad+h\left(y-y_{0}\right) \exp \left[-p_{i}^{\Sigma}\left(y-y_{0}\right)\right]\right\},
\end{aligned}
$$


with $h(y)$ being the Heaviside function. The characteristic momenta $p_{i}^{\Sigma}$ and $p_{j}^{\Sigma}$ are also given by Eq. (31). The behavior of the EF $\eta_{i j}(x, y)$ just near the interdomain boundary, at $\left|y-y_{0}\right|<\left(p_{i, j}^{\Sigma}\right)^{-1}$, is determined by the electrostatic potential $\mathbb{S}$ generated by the QL step (which is abbreviated to SP from "step potential"). The energy spectrum of the inner edge state is gapless and can be presented around the point $k_{x}=0$ by the linear-in- $k_{x}$ dispersion branches

$$
\epsilon^{\Sigma}\left(k_{x}\right)=\epsilon_{0}^{\Sigma}(S)+\operatorname{sgn}(\Sigma)|\mathcal{A}| k_{x},
$$

where the Dirac point is shifted with respect to the middle of the gap $2 \Delta_{0 i}^{\Sigma}$. The value and sign of the shift is mostly affected by SP strength, but the dependence $\epsilon_{0}^{\Sigma}(S)$ is rather complicated.

As noted above, not every QL step originates a domain wall. Nevertheless, the QL step, being placed at $y_{0}$ inside the region of either the topological phase or the trivial one, can cause a bound state with EF approximated far from the step by

$$
\zeta_{i}^{\Sigma}(x, y) \sim \exp \left(i k_{x} x\right) \exp \left(-p_{i}^{\Sigma}\left|y-y_{0}\right|\right),
$$

where $p_{i}^{\Sigma}$ is also given by Eq. (31). The spectrum of the stepinduced state is gapped:

$$
v^{\Sigma}\left(k_{x}\right)=v_{0}^{\Sigma}(S) \pm \sqrt{\left[\gamma^{\Sigma}(S)\right]^{2}+|\mathcal{A}|^{2} k_{x}^{2}} .
$$

One can show that the energy shift $v_{0}^{\Sigma}(S)$ and the gap $2 \gamma^{\Sigma}(S)$ decrease reciprocally proportionally to the SP strength when it is large enough. The inner states $\zeta_{i}^{\Sigma}(x, y)$ are ubiquitous for the rough interface as they appear at each QL step regardless of the band topology of the phase $i$. As a result, the inner states are not robust to dissipation. Nevertheless, because of the nearly Dirac spectrum (36), they can show low-loss ballistic transport in clean enough samples. The thorough analysis of the experimental data accompanied by numerical simulation accomplished in Ref. [53] reveals the emergence of bound 1D edge states near steps at the surface of $\mathrm{Bi}_{2} \mathrm{Se}_{3}$.

Due to axial rotation symmetry of the Hamiltonian (11), the generic forms of the outer or inner edge state spectrum versus the momentum along a boundary [Eqs. (32), (34), and (36)] and the EF spatial profile [Eqs. (30), (33), and (35)] will be the same for any orientation of the side surface and the QL step, respectively. In the tetradymite films, the QL steps are oriented with respect to each other according to the threefold crystal symmetry. In Ref. [54] the SP associated with the QL step at the TI surface is found as a repulsive potential with $S=3.8 \mathrm{eV}$ applied to each atom along the step path. At the TI/FMNI interface the dangling bonds of the constituents at the QL step are reconnected, which leads probably to renormalized value $S$. The OEP $\mathbb{V}$ is of another nature: it is determined by the termination and atomic structure of the side surface separating the TI film from the topologically trivial surroundings (see Fig. 1). In the interface plane, the decay length of the inner and outer edge states is the function of the thickness $\sim\left[\operatorname{Re}\left(p_{i}\right)\right]^{-1} \simeq l \exp \left(-2 q_{0} l\right)$. The topologically protected edge states also exhibit the pseudospin to momentum locking. One should note that a behavior of the outer and inner edge states is highly sensitive to the signs of $\Delta_{0}^{\Sigma}$ and $b^{\Sigma}$. The simultaneous change of signs of $b^{\Sigma}$ and $\Delta_{0}^{\Sigma}$ leads to the switching of the polarization of the state.
The topologically protected outer edge state $\vartheta_{i}^{\Sigma}(x, y)$ is defined for an infinite straight side surface. Analogously, the inner edge state $\eta_{i j}^{\Sigma}(x, y)$ is specified in the ideal case of an infinite straight boundary between two phases with distinct topological indices. In the heterostructure setups prepared for magnetotransport measurements, the TI film is always bounded in the $(x, y)$ plane, for example, being patterned into the Hall bar geometry. When the interfaces of the TI/FMNI trilayer are ideally plane, i.e., without roughness, the thin bounded film is the single-phase domain $i$ (in the topological sense), in which the numbers $\left\{C_{i}^{\Uparrow}, C_{i}^{\Downarrow}\right\}$ are unambiguously determined by the film thickness according to the diagram in Fig. 2. In this case, the QSHE phase or the mixed QAHE+QSHE phase are manifested through the helical or chiral outer edge states $\vartheta_{i}^{\Sigma}(x, y)$ under the proper position of the chemical potential $\mu$. The step-and-terrace morphology of the TI/FMNI interfaces in real samples brings about the irregular net of the inner edge states, making the topological transport profoundly more complex. Under the stipulation that the characteristic interphase distance $L_{0}$ is much larger than the roughness amplitude of a few QLs, i.e., $L_{0} \gg h_{\mathrm{QL}}$, the film can also be imagined as the single-phase domain. However, in this situation, the outer edge states $\vartheta_{i}^{\Sigma}(x, y)$ interfere with the inner edge states $\zeta_{i}{ }^{\Sigma}(x, y)$ residing at the QL steps which adjoin the side surfaces, as schematically depicted in Fig. 5. Thus, both topologically protected channels and unprotected ones are involved parallelly in the conductivity along the outer edge of the single-phase film. As a consequence, the perfect quantization of the Hall conductance of the sample can be undermined.

When the characteristic interphase interval $L_{0}$ becomes comparable with the QL height, the phase decomposition happens within the TI film. If $L_{0} \gtrsim h_{\mathrm{QL}}$, the film acquires multidomain spatial structure where the trivial, QSHE, and mixed QAHE+QSHE phase fractions coexist. On the domain structure map (Fig. 5), one can mark out three geometrically distinct types of spatial regions, inside which the numbers $\left\{C^{\Uparrow}(x, y), C^{\Downarrow}(x, y)\right\}$ are invariable. The first type consists of macrodomain regions (the region MD in Fig. 5) of the terrace width $\sim \mathcal{L}$, or larger, which can in principle span the film from one side surface to opposite side surface. The second type, abbreviated to ID (island domain), includes isolated islandlike regions of one phase surrounded by regions of other phases. These regions do not reach the side surface of the film, therefore, their inner edges encircle the islands, having no contribution to transport. The third are domain regions of finite area of the order of or less than $\mathcal{L}$ adjoining the side surface of the film, as depicted and denoted by AD (adjoining domain) in Fig. 5. Each of such regions is bordered by both the inner and outer edges.

As long as the regions of the first and third types exist in the multidomain film, the charge current channel, squeezed close to the film side surface, is decomposed to segments of the outer edges of length $\leqslant \mathcal{L}$. The charge carrier is assumed to propagate along the segment with a sufficiently long scattering length, i.e., ballistically. However, these segments host the dissimilar states $\vartheta_{i}^{\Sigma}(x, y)$. For the third type domain region, the current is divided between the inner and outer edges bordering this domain. As for the dispersion laws, the Dirac point of the outer edge state $\vartheta_{i}^{\Sigma}(x, y)$ with the energy $\varepsilon_{0}^{\Sigma}(V)$ 


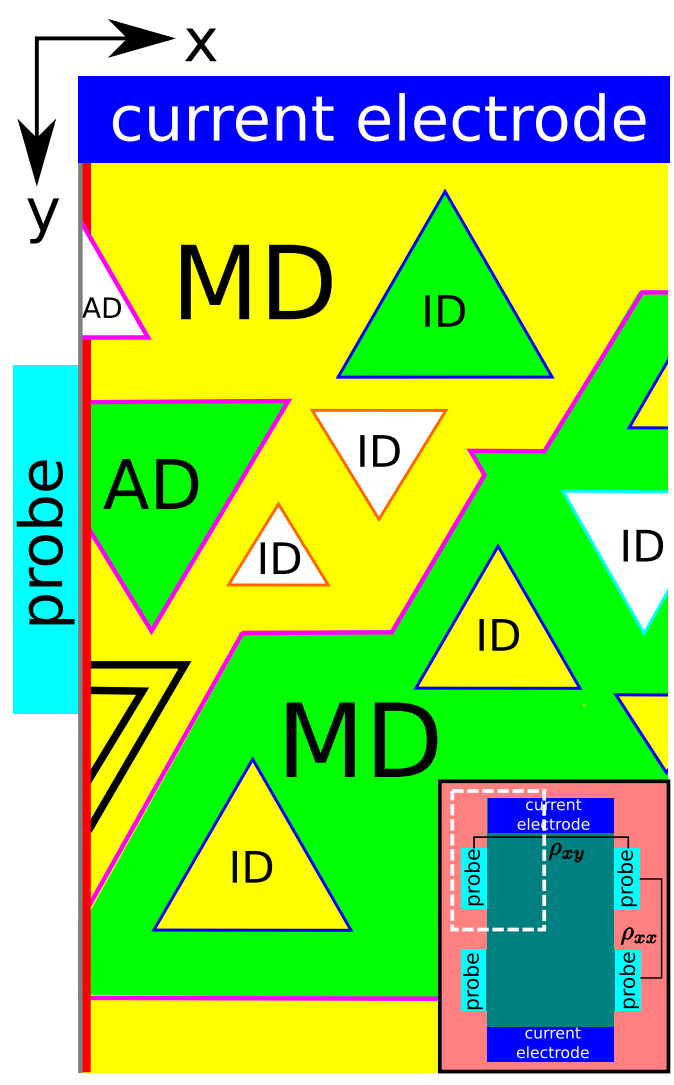

FIG. 5. Schematic illustration of domain structure of the TI thin film sandwiched between the FMNI slabs. The interface morphology is represented by terraces separated by QL steps oriented along hexagonal axes consistently with the threefold symmetry of tetradymite semiconductors. The sketch shows a fragment of the trilayer patterned into the Hall bar geometry (see the inset), which includes the current drain contact and one of probes. The interface roughness in the TI film is expected to induce domains of topologically different phases along the interface plane $(x, y)$, which are separated by the domain walls hosting inner conducting channels. Topography of domain structure can be represented by islands (labeled by "ID'), regions adjoining to side surface (labeled by "AD") and macrodomains (labeled by "MD"). See text for details. Here, one observes a particular configuration consisting of three topologically distinct insulating phases: the trivial phase (white regions); QSHE phase (green regions); the mixed QAHE+QSHE phase (yellow regions). For instance, in accordance with the phase diagram in Fig. 2, this configuration might occur in the TI film with the thickness fluctuating in the range of $2.5 \lesssim \widetilde{l} \lesssim 4$ and $\widetilde{\omega} \simeq 0.15$. Outer edge channel appearing along the side surface is indicated by the thick red line.

[Eq. (32)] is driven by OEP, while that of the inner edge state $\eta_{i j}^{\Sigma}(x, y)$ with the energy $\epsilon_{0}^{\Sigma}(S)$ [Eq. (34)] is driven by SP, where OEP and SP have different physical derivation. Such difference between the positions $\varepsilon_{0}^{\Sigma}(V)$ and $\epsilon_{0}^{\Sigma}(S)$ rather leads to the electron scattering at a vertex, where the inner edge meets the outer edge (Fig. 5). Apart from that, the aforesaid effect of the unprotected inner states $\zeta_{i}^{\Sigma}(x, y)$ (shown by black lines near the side surface) on the outer states still retains. Under the condition $L_{0}<h_{\mathrm{QL}}$, almost each QL step becomes the phase boundary in the $(x, y)$ plane and the domain size is $\sim \mathcal{L}$. Thus, the roughness randomness results in pronounced nanoscale spatial fluctuations of the energy spectrum, topological robustness, pseudospin polarization, and the number of propagating modes of the edge states. Remember, in the standard Hall bar devices the distance between the terminal and probe contacts is of the order of $\sim 0.1-1 \mathrm{~mm}$, which is much larger than the characteristic terrace width $\mathcal{L}$ of few tens of nanometers. Therefore, owing to the irregular multidomain geometry of the film imposed by the interface roughness, the topological protection scale (i.e., the distance on which the carriers in an edge channel propagate without backscattering) in the TI/FMNI heterostructures is much shorter than the channel length of the Hall bar device. As a result, the measured conductivity can display remarkable deviation from the expected quantized value.

Within the framework of our analytic approach, one can suggest the way to avoid the destructive influence of the interface roughness on the spin-resolved electron transport in the heterostructures. The interphase interval $L_{0}$ is dependent on the 3D TI bulk band structure parameters. It is understood, with the increasing parameter $\lambda$, the term $\Delta^{\Sigma}(\kappa)$ as a function of $l$ oscillates slower and becomes monotonically decreasing at $\lambda>1$. Then, the moderate interface roughness practically does not affect the quantization of the Hall conductivity. Noteworthy, the condition $\lambda>1$ associates with the relatively small bulk band gap since $\lambda \sim \Xi_{0}^{-1}$.

In accordance with the foregoing, the transition to the quantum Hall conductivity plateau state can be considered as a percolation of the network of the outer and inner edge channels running along the side surfaces of a sample. It is relevant to note here that a gradual saturation of Hall conductivity to $\frac{e^{2}}{h}$ has been recently observed in thin films of $(\mathrm{Bi}, \mathrm{Sb})_{2} \mathrm{Te}_{3}$ sandwiched by 2 -nm-thick $\mathrm{Cr}$-doped $(\mathrm{Bi}, \mathrm{Sb})_{2} \mathrm{Te}_{3}$ layers under magnetization rotation to the out-of-plane direction [55].

The underlying mechanism for breaking the Hall conductivity quantization is improper when the roughness is so strong that the size of the phase domains becomes comparable with the EF extension $\sim p_{i}^{-1}$. In this case, a more sophisticated treatment is required, which takes into consideration the quantum effects.

\section{DISCUSSION}

In the context of the obtained results, it is worth discussing the specifics of the TI/FMNI heterostructure application for realization of QAHE as compared with the approach based on the use of the FMTI film with uniform magnetic doping. One can notice a certain similarity between the phase diagram of the FMNI/TI/FMNI trilayer and that of the NI/FMTI/NI trilayer [45], although the underlying physical mechanisms to generate the exchange field in the TI film differ. In both cases, in the parameter space $(\omega, l)$, there are trivial, QSHE, and mixed QAHE+QSHE phases, and the latter exists at the relatively large exchange splitting. The heterostructure approach provides a control of the intrinsic Hall response without facing the known problems of magnetic impurity doping in the spacer [6-9]. From the above analysis, it is clear that there is no necessity to expand the FM order on whole TI film to drive it to the QAHE regime, as it usually happens in the case of FMTI with dilute magnetic impurities [12-16]. At 
that, the TI/FMNI interface properties are essential for realization of the proximity-induced QAHE regime. As far as is known [40,41], the significant charge transfer at the TI/FMNI interface, accompanied by the band bending, dominates the magnetic proximity effect. Our model catches that the induced exchange splitting of the states in the TI film is suppressed by potential scattering at the interface [see Eq. (20)]. The DFT simulations show that in both the $\mathrm{MnSe} / \mathrm{Bi}_{2} \mathrm{Se}_{3}$ [40] and $\mathrm{EuS} / \mathrm{Bi}_{2} \mathrm{Se}_{3}$ [23] heterostructures, the proximity effect is weak, as a result, the exchange splitting of the Dirac point is small, about a few meV. Nevertheless, to generate the largest spin splitting of the topological states, a system should be arranged in such a manner that the maximum of the electron probability density coincides with that of the magnetization distribution. For example, the structure with the magnetic modulation doped TI film demonstrates a substantial dependence of the exchange splitting on the distance between the ultrathin FM insertion and the TI/NI interface [19,20].

\section{CONCLUSION}

In conclusion, the problem of QAHE in the TI/FMNI heterostructures under the magnetic proximity effect is considered within the framework of the analytical scheme. We treat the influence of the adjacent FMNI slab on the topologically relevant electron states in the TI film through the corresponding natural boundary conditions. It is emphasized that the proximity-induced exchange splitting of the lowenergy interface electron states in the TI film depends essentially on the potential component of IP. We found, by the example of the FMNI/TI/FMNI trilayer, that the interface states in the TI film and, as a consequence, the Hall response can be manipulated by changing the conditions at the interfaces and the film thickness as well as the band structure parameters of the TI material. We depict the phase diagram for the trilayer demonstrating the quantum transitions between topologically distinct phases driven by the key factors of the system. We show how the quantized regimes of spin or anomalous Hall response can be manipulated by changing the TI film thickness or the proximity-induced exchange splitting. Thereby, we elucidate a necessary condition for the existence of the electron topological phase manifesting QAHE. We also describe the evolution of the energy spectrum and the Berry curvature under the transition between different phases of the quantized Hall conductivity. Moreover, we infer how the behavior of the intrinsic Hall conductivity is influenced by the TI/FMNI interface roughness inherent to the realistic heterostructures. Our predications offer deeper insight into the quantum phenomena in the TI/FMNI heterostructures, which will help to interpret magnetotransport measurements and intentionally design the layered structure providing the QAHE regime realization at practically relevant temperatures.

\section{ACKNOWLEDGMENTS}

We acknowledge partial support from the Basque Country Government, Departamento de Educación, Universidades e Investigación (Grant No. IT-756-13), the Spanish Ministry of Science and Innovation (Grant No. FIS2016-75862-P), the Tomsk State University Academic D.I. Mendeleev Fund Program (Grant No. 8.1.05.2015), Saint Petersburg State University (Grant No. 15.61.202.2015), Russian Science Foundation (Grant No. 18-12-000169), and Russian Foundation for Basic Research (Grant No. 16-02-00024). Numerical calculations were partly performed using computational resources provided by Resource Center "Computer Center of SPbU" and the SKIF-Cyberia supercomputer at the National Research Tomsk State University.
[1] I. Žutić, J. Fabian, and S. Das Sarma, Spintronics: Fundamentals and applications, Rev. Mod. Phys. 76, 323 (2004).

[2] J. Sinova, S. O. Valenzuela, J. Wunderlich, C. H. Back, and T. Jungwirth, Spin Hall effects, Rev. Mod. Phys. 87, 1213 (2015).

[3] M. Z. Hasan and C. L. Kane, Colloquium: Topological insulators, Rev. Mod. Phys. 82, 3045 (2010).

[4] X.-L. Qi and S.-C. Zhang, Topological insulators and superconductors, Rev. Mod. Phys. 83, 1057 (2011).

[5] N. Nagaosa, J. Sinova, S. Onoda, A. H. MacDonald, and N. P. Ong, Anomalous Hall effect, Rev. Mod. Phys. 82, 1539 (2010).

[6] C.-Z. Chang and M. Li, Quantum anomalous Hall effect in timereversal-symmetry breaking topological insulators, J. Phys.: Condens. Matter 28, 123002 (2016).

[7] X. Kou, Y. Fan, M. Lang, P. Upadhyaya, and K. L. Wang, Magnetic topological insulators and quantum anomalous hall effect, Solid State Commun. 215-216, 34 (2015).

[8] H. Weng, R. Yu, X. Hu, X. Dai, and Z. Fang, Quantum anomalous Hall effect and related topological electronic states, Adv. Phys. 64, 227 (2015).

[9] C.-X. Liu, S.-C. Zhang, and X.-L. Qi, The Quantum Anomalous Hall Effect: Theory and Experiment, Annu. Rev. Condens. Matter Phys. 7, 301 (2016).
[10] A. M. Essin, J. E. Moore, and D. Vanderbilt, Magnetoelectric Polarizability and Axion Electrodynamics in Crystalline Insulators, Phys. Rev. Lett. 102, 146805 (2009).

[11] D. Xiao, M.-C. Chang, and Q. Niu, Berry phase effects on electronic properties, Rev. Mod. Phys. 82, 1959 (2010).

[12] C.-Z. Chang, J. Zhang, X. Feng, J. Shen, Z. Zhang, M. Guo, K. Li, Y. Ou, P. Wei, L.-L. Wang, Z.-Q. Ji, Y. Feng, S. Ji, X. Chen, J. Jij, X. Dai, Z. Fang, S.-C. Zhang, K. He, Y. Wang et al., Experimental observation of the quantum anomalous Hall effect in a magnetic topological insulator, Science 340, 167 (2013).

[13] C.-Z. Chang, W. Zhao, D. Y. Kim, H. Zhang, B. A. Assaf, D. Heiman, S.-C. Zhang, C. Liu, M. H. W. Chan, and J. S. Moodera, High-precision realization of robust quantum anomalous Hall state in a hard ferromagnetic topological insulator, Nat. Mater. 14, 473 (2014).

[14] A. J. Bestwick, E. J. Fox, X. Kou, L. Pan, K. L. Wang, and D. Goldhaber-Gordon, Precise Quantization of the Anomalous Hall Effect Near Zero Magnetic Field, Phys. Rev. Lett. 114, 187201 (2015).

[15] C.-Z. Chang, W. Zhao, D. Y. Kim, P. Wei, J. K. Jain, C. Liu, M. H. W. Chan, and J. S. Moodera, Zero-Field Dissipationless Chiral Edge Transport and the Nature of Dissipation in the 
Quantum Anomalous Hall State, Phys. Rev. Lett. 115, 057206 (2015).

[16] M. Winnerlein, S. Schreyeck, S. Grauer, S. Rosenberger, K. M. Fijalkowski, C. Gould, K. Brunner, and L. W. Molenkamp, Epitaxy and Structural Properties of $(\mathrm{V}, \mathrm{Bi}, \mathrm{Sb})_{2} \mathrm{Te}_{3}$ Layers Exhibiting the Quantum Anomalous Hall Effect, Phys. Rev. Mater. 1, 011201 (2017).

[17] I. Lee, C. K. Kim, J. Lee, S. J. L. Billinge, R. Zhong, J. A. Schneeloch, T. Liu, T. Valla, J. M. Tranquada, G. Gu, and J. C. S. Davis, Imaging Dirac-mass disorder from magnetic dopant atoms in the ferromagnetic topological insulator $\mathrm{Cr}_{x}\left(\mathrm{Bi}_{0.1} \mathrm{Sb}_{0.9}\right)_{2-x} \mathrm{Te}_{3}$, Proc. Natl. Acad. Sci. USA 112, 1316 (2015).

[18] J. G. Checkelsky, R. Yoshimi, A. Tsukazaki, K. S. Takahashi, Y. Kozuka, J. Falson, M. Kawasaki, and Y. Tokura, Trajectory of the anomalous Hall effect towards the quantized state in a ferromagnetic topological insulator, Nat. Phys. 10, 731 (2014).

[19] M. Mogi, R. Yoshimi, A. Tsukazaki, K. Yasuda, Y. Kozuka, K. S. Takahashi, M. Kawasaki, and Y. Tokura, Magnetic modulation doping in topological insulators toward highertemperature quantum anomalous Hall effect, Appl. Phys. Lett. 107, 182401 (2015).

[20] K. N. Okada, Y. Takahashi, M. Mogi, R. Yoshimi, A. Tsukazaki, K. S. Takahashi, N. Ogawa, M. Kawasaki, and Y. Tokura, Terahertz spectroscopy on Faraday and Kerr rotations in a quantum anomalous Hall state, Nat. Commun. 7, 12245 (2016)

[21] P. Wei, F. Katmis, B. A. Assaf, H. Steinberg, P. Jarillo-Herrero, D. Heiman, and J. S. Moodera, Exchange-Coupling-Induced Symmetry Breaking in Topological Insulators, Phys. Rev. Lett. 110, 186807 (2013).

[22] Q. I. Yang, M. Dolev, L. Zhang, J. Zhao, A. D. Fried, E. Schemm, M. Liu, A. Palevski, A. F. Marshall, S. H. Risbud, and A. Kapitulnik, Emerging weak localization effects on a topological insulator-insulating ferromagnet $\left(\mathrm{Bi}_{2} \mathrm{Se}_{3}-\mathrm{EuS}\right)$ interface, Phys. Rev. B 88, 081407(R) (2013).

[23] S. V. Eremeev, V. N. Men'shov, V. V. Tugushev, and E. V. Chulkov, Interface induced states at the boundary between a $3 \mathrm{D}$ topological insulator $\mathrm{Bi}_{2} \mathrm{Se}_{3}$ and a ferromagnetic insulator $\mathrm{EuS}$, J. Magn. Magn. Mater. 383, 30 (2015).

[24] C. Lee, F. Katmis, P. Jarillo-Herrero, J. S. Moodera, and N. Gedik, Direct measurement of proximity-induced magnetism at the interface between a topological insulator and a ferromagnet, Nat. Commun. 7, 12014 (2016).

[25] F. Katmis, V. Lauter, F. S. Nogueira, B. A. Assaf, M. E. Jamer, P. Wei, B. Satpati, J. W. Freeland, I. Eremin, D. Heiman, P. Jarillo-Herrero, and J. S. Moodera, A high-temperature ferromagnetic topological insulating phase by proximity coupling, Nature (London) 533, 513 (2016).

[26] J. Kim, K.-W. Kim, H. Wang, J. Sinova, and R. Wu, Understanding the Giant Enhancement of Exchange Interaction in $\mathrm{Bi}_{2} \mathrm{Se}_{3} / \mathrm{EuS}$ Heterostructures, Phys. Rev. Lett. 119, 027201 (2017).

[27] M. Li, Q. Song, W. Zhao, J. A. Garlow, T.-H. Liu, L. Wu, Y. Zhu, J. S. Moodera, M. H. W. Chan, G. Chen, and C.-Z. Chang, Dirac-electron-mediated magnetic proximity effect in topological insulator/magnetic insulator heterostructures, Phys. Rev. B 96, 201301(R) (2017).
[28] L. D. Alegria, H. Ji, N. Yao, J. J. Clarke, R. J. Cava, and J. R. Petta, Large anomalous Hall effect in ferromagnetic insulatortopological insulator heterostructures, Appl. Phys. Lett. 105, 053512 (2014).

[29] H. Ji, R. A. Stokes, L. D. Alegria, E. C. Blomberg, M. A. Tanatar, A. Reijnders, L. M. Schoop, T. Liang, R. Prozorov, K. S. Burch, N. P. Ong, J. R. Petta, and R. J. Cava, A ferromagnetic insulating substrate for the epitaxial growth of topological insulators, J. Appl. Phys. 114, 114907 (2013).

[30] C. Tang, C.-Z. Chang, G. Zhao, Y. Liu, Z. Jiang, C.-X. Liu, M. R. McCartney, D. J. Smith, T. Chen, J. S. Moodera, and J. Shi, Above 400-K robust perpendicular ferromagnetic phase in a topological insulator, Sci. Adv. 3, e1700307 (2017).

[31] W. M. Yang, S. Yang, Q. Zhang, Y. Xu, S. Shen, J. Liao, J. Teng, C. Nan, L. Gu, Y. Sun, K. Wu, and Y. Li, Proximity effect between a topological insulator and a magnetic insulator with large perpendicular anisotropy, Appl. Phys. Lett. 105, 092411 (2014).

[32] J. S. Lee, A. Richardella, R. D. Fraleigh, C.-x. Liu, W. Zhao, and N. Samarth, Engineering the breaking of time-reversal symmetry in gate-tunable hybrid ferromagnet/topological insulator heterostructures, npj Quantum Materials 3, 51 (2018).

[33] X.-L. Qi, T. L. Hughes, and S.-C. Zhang, Topological field theory of time-reversal invariant insulators, Phys. Rev. B 78, 195424 (2008).

[34] R. Yu, W. Zhang, H.-J. Zhang, S.-C. Zhang, X. Dai, and Z. Fang, Quantized anomalous Hall effect in magnetic topological insulators, Science 329, 61 (2010).

[35] S.-Q. Shen, Topological Insulators (Springer, Berlin, 2012).

[36] H. J. Zhang, C. X. Liu, X. L. Qi, X. Dai, Z. Fang, and S. C. Zhang, Topological insulators in $\mathrm{Bi}_{2} \mathrm{Se}_{3}, \mathrm{Bi}_{2} \mathrm{Te}_{3}$ and $\mathrm{Sb}_{2} \mathrm{Te}_{3}$ with a single Dirac cone on the surface, Nat. Phys. 5, 438 (2009).

[37] V. N. Men'shov, V. V. Tugushev, T. V. Menshchikova, S. V. Eremeev, P. M. Echenique, and E. V. Chulkov, Modelling nearsurface bound electron states in a $3 \mathrm{D}$ topological insulator: analytical and numerical approaches, J. Phys.: Condens. Matter 26, 485003 (2014).

[38] V. N. Men'shov, V. V. Tugushev, S. V. Eremeev, P. M. Echenique, and E. V. Chulkov, Band bending driven evolution of the bound electron states at the interface between a threedimensional topological insulator and a three-dimensional normal insulator, Phys. Rev. B 91, 075307 (2015).

[39] V. N. Men'shov, V. V. Tugushev, and E. V. Chulkov, Interface induced states at the boundary between a 3D topological insulator and a normal insulator, JETP Lett. 97, 258 (2013).

[40] S. V. Eremeev, V. N. Men'shov, V. V. Tugushev, P. M. Echenique, and E. V. Chulkov, Magnetic proximity effect at the three-dimensional topological insulator/magnetic insulator interface, Phys. Rev. B 88, 144430 (2013).

[41] V. N. Men'shov, V. V. Tugushev, S. V. Eremeev, P. M. Echenique, and E. V. Chulkov, Magnetic proximity effect in the three-dimensional topological insulator/ferromagnetic insulator heterostructure, Phys. Rev. B 88, 224401 (2013).

[42] V. N. Men'shov, I. A. Shvets, V. V. Tugushev, and E. V. Chulkov, Intrinsic spin Hall conductivity in three-dimensional topological insulator/normal insulator heterostructures, Phys. Rev. B 96, 075302 (2017). 
[43] V. N. Men'shov, V. V. Tugushev, and E. V. Chulkov, Spin Hall Conductivity in Three-Dimensional Topological Insulator/Normal Insulator Heterostructures, JETP Lett. 102, 754 (2015).

[44] H.-Z. Lu, W.-Y. Shan, W. Yao, Q. Niu, and S.-Q. Shen, Massive Dirac fermions and spin physics in an ultrathin film of topological insulator, Phys. Rev. B 81, 115407 (2010).

[45] V. N. Men'shov, V. V. Tugushev, and E. V. Chulkov, Anomalous Hall and spin Hall conductivities in three-dimensional ferromagnetic topological insulator/normal insulator heterostructures, Europhys. Lett. 114, 37003 (2016).

[46] V. N. Men'shov, V. V. Tugushev, and E. V. Chulkov, Quantum anomalous Hall effect in magnetically modulated topological insulator/normal insulator heterostructures, JETP Lett. 104, 453 (2016).

[47] V. V. Tugushev, V. N. Men'shov, I. A. Shvets, and E. V. Chulkov, Quantum anomalous Hall conductivity in 3D magnetic topological insulator/normal insulator heterostructures, J. Magn. Magn. Mater. 459, 335 (2018).

[48] N. V. Tarakina, S. Schreyeck, M. Luysberg, S. Grauer, C. Schumacher, G. Karczewski, K. Brunner, C. Gould, H. Buhmann, R. E. Dunin-Borkowski, and L. W. Molenkamp, Suppressing twin formation in $\mathrm{Bi}_{2} \mathrm{Se}_{3}$ thin films, Adv. Mater. Interfaces 1, 1400134 (2014).

[49] Y. H. Liu, C. W. Chong, W. C. Chen, J. C. A. Huang, C.-M. Cheng, K.-D. Tsuei, Z. Li, H. Qiu, and V. V. Marchenkov,
Robust topological insulator surface state in MBE grown $\left(\mathrm{Bi}_{1-x} \mathrm{Sb}_{x}\right)_{2} \mathrm{Se}_{3}$, arXiv:1611.08395.

[50] I. A. Nechaev and E. E. Krasovskii, Relativistic kp Hamiltonians for centrosymmetric topological insulators from $a b$ initio wave functions, Phys. Rev. B 94, 201410(R) (2016).

[51] J. Linder, T. Yokoyama, and A. Sudbø, Anomalous finite size effects on surface states in the topological insulator $\mathrm{Bi}_{2} \mathrm{Se}_{3}$, Phys. Rev. B 80, 205401 (2009).

[52] C. X. Liu, H. J. Zhang, B. H. Yan, X. L. Qi, T. Frauenheim, X. Dai, Z. Fang, and S. C. Zhang, Oscillatory crossover from two-dimensional to three-dimensional topological insulators, Phys. Rev. B 81, 041307(R) (2010).

[53] N. I. Fedotov and S. V. Zaitsev-Zotov, Experimental search for one-dimensional edge states at surfacesteps of the topological insulator $\mathrm{Bi}_{2} \mathrm{Se}_{3}$ : Distinguishing between effects and artifacts, Phys. Rev. B 95, 155403 (2017).

[54] Y. Xu, J. Chiu, L. Miao, H. He, Z. Alpichshev, A. Kapitulnik, R. R. Biswas, and L. A. Wray, Disorder enabled band structure engineering of a topological insulator surface, Nat. Commun. 8, 14081 (2017).

[55] M. Kawamura, M. Mogi, R. Yoshimi, A. Tsukazaki, Y. Kozuka, K. S. Takahashi, M. Kawasaki, and Y. Tokura, Topological quantum phase transition in magnetic topological insulator upon magnetization rotation, Phys. Rev. B 98, 140404(R) (2018). 